\title{
Sensitivities of Baseline Isolates and Boscalid-Resistant Mutants of Alternaria alternata from Pistachio to Fluopyram, Penthiopyrad, and Fluxapyroxad
}

\author{
H. F. Avenot, Department of Plant Pathology, University of California-Davis, Kearney Agricultural Research and Extension Center, \\ Parlier 93648; H. van den Biggelaar, Laboratory of Phytopathology, Wageningen University, 6708 PB Wageningen, The Netherlands; \\ D. P. Morgan, Department of Plant Pathology, University of California-Davis, Kearney Agricultural Research and Extension Center, \\ Parlier; J. Moral, Departmento de Agronomía, ETSIAM, Universidad de Córdoba, Campus de Rabanales, Edif. C4, 14071 Córdoba, \\ Spain; M. Joosten, Laboratory of Phytopathology, Wageningen University; and T. J. Michailides, Department of Plant Pathology, \\ University of California-Davis, Kearney Agricultural Research and Extension Center, Parlier
}

\begin{abstract}
Avenot, H. F., van den Biggelaar, H., Morgan, D. P., Moral, J., Joosten, M., and Michailides, T. J. 2014. Sensitivities of baseline isolates and boscalid-resistant mutants of Alternaria alternata from pistachio to fluopyram, penthiopyrad, and fluxapyroxad. Plant Dis. 98:197-205.

Resistance of Alternaria alternata to boscalid, the first succinate dehydrogenase inhibitor (SDHI) fungicide labeled on pistachio, has become a common occurrence in California pistachio orchards and affects the performance of this fungicide. In this study, we established the baseline sensitivities of A. alternata to the new SDHIs fluopyram, fluxapyroxad, and penthiopyrad and assessed their cross resistance patterns with boscalid. Examination of the effective fungicide concentration that inhibits mycelial growth to $50 \%$ relative to the control $\left(\mathrm{EC}_{50}\right)$ for 50 baseline isolates revealed that the majority were sensitive to boscalid, penthiopyrad, fluopyram, and fluxapyroxad. Analysis of $\mathrm{EC}_{50}$ values for boscalid for $117 \mathrm{~A}$. alternata isolates originating from boscalid-exposed orchards showed that $44,3,1$, and 69 isolates had sensitive, reduced sensitivity, moderately resistant, and highly resistant boscalid phenotypes, respectively. Molecular investigation of the occurrence of known $S D H$ mutations showed that, among the 69 isolates highly resistant to boscalid, 44, 2, 14, and 1 isolates possessed the

mutations leading to the H277Y, H277R, H134R, and H133R amino acid substitutions in AaSDHB, AaSDHB, AaSDHC, and AaSDHD subunits, respectively. Some $S D H B$ or $S D H C$ mutants displayed highly sensitive, sensitive, or reduced sensitivity phenotypes toward penthiopyrad or fluxapyroxad, whereas other had low, moderate, or high levels of resistance to these fungicides. In contrast, all the $S D H B$ mutants were sensitive to fluopyram, while 10,5 , and $1 S D H C$ mutants had sensitive, reduced sensitivity, and moderately resistant fluopyram phenotypes, respectively. The SDHD mutant had reduced sensitivity to fluopyram and penthiopyrad but was highly resistant to fluxapyroxad. The discrepancies of cross-resistance patterns between SDHIs suggest that their binding sites in complex II may differ slightly and that additional mechanisms of resistance to these compounds are likely involved. Ultimately, the findings of this study should lead to the rational and sustained deployment of new SDHIs in Alternaria late blight spray programs.
\end{abstract}

Alternaria late blight (ALB), caused by Alternaria alternata, $A$. tenuissima, and $A$. arborescens, is now considered the most destructive disease of pistachio in California. The disease affects foliage and fruit and is an annual production concern for pistachio growers (20). On foliage, it can be recognized by the development of large necrotic lesions, and multiple, expanding lesions eventually consume the whole leaf. The lesions are black in the center due to the production of many spores and are surrounded by a chlorotic halo. Under optimal conditions for the disease, the fungus can defoliate a tree in late summer and autumn (20). On fruit, it is characterized by small necrotic lesions surrounded by a red halo. These are located on the hull of immature nuts. When the nuts develop, one or two lesions can penetrate and decay the hull, resulting in shell staining of the nut underneath. The staining of the shell and colonization of the kernel result in a reduction of the nut quality (20). Although cultural practices such as irrigation management and pruning to increase air movement and decrease air humidity in the orchard can help reduce ALB, the use of multiple fungicide applications is essential to achieve adequate disease control.

Corresponding authors: H. Avenot, E-mail: hfavenot@ucanr.edu, and T. Michailides, E-mail: tjmichailides@ucanr.edu

Current address of H. van den Biggelaar: Nickerson-Zwaan B.V., Schanseind 27, 4921 PM Made, The Netherlands.

Accepted for publication 19 July 2013.

http://dx.doi.org/10.1094/PDIS-04-13-0459-RE

(c) 2014 The American Phytopathological Society
Over the last 10 years, respiration-inhibiting fungicides, including quinone outside inhibitors (QoIs, registered in 2000) and the succinate dehydrogenase inhibitor (SDHI) boscalid (registered in 2003), have been vital components in ALB fungicide spray programs. However, due to their single mode of action, the usefulness of available products in these classes has been eliminated by the rapid development of Alternaria populations resistant to them. Azoxystrobin (Abound), the first QoI registered on pistachio, was a highly effective fungicide in controlling ALB on pistachio. However, the development of azoxystrobin-resistant Alternaria isolates carrying a G143A amino acid substitution in the cytochrome bc1 protein has affected the effectiveness of this fungicide and that of other QoI compounds that were subsequently introduced due to cross resistance $(4,14-18)$. The introduction of the SDHI fungicide boscalid, effective against a wide spectrum of pathogens, offered new possibilities for the control of many fungal diseases in various crops (24), including ALB. SDHIs have a unique mode of action and block the fungal respiration process by binding to the ubiquinone reduction site of complex II of the respiratory chain, also known as succinate dehydrogenase $(\mathrm{SDH})$ or succinate:ubiquinone oxido-reductase $(3,11,24)$.

Boscalid was introduced on pistachio in the premixture product Pristine, along with the strobilurin pyraclostrobin, and provided high efficiency in controlling ALB even in orchards with widespread resistance to QoIs (4). However, strains of Alternaria resistant to boscalid were detected within two seasons after registration and commercial use of Pristine on pistachio $(1,4)$. Very rapidly, resistance to boscalid became widespread in Alternaria populations in several pistachio orchards, thus seriously affecting its performance $(2,3)$. Investigations of the molecular mechanism of boscalid resistance in Alternaria spp. showed that resistance was 
associated with several different mutations in the A. alternata $S D H$ $B, C$, and $D$ genes, encoding the iron sulfur subunit (AaSDHB) and two membrane subunits (AaSDHC and AaSDHD) of succinate dehydrogenase $(5,6)$.

Because pistachio growers continue to rely on fungicides to effectively manage ALB and, because of the ability of Alternaria spp. of pistachio to develop resistance to site-specific fungicides, there is a continuous need for new and more effective active ingredients (8) to control this disease. New active ingredients belonging to the SDHI group have now been discovered and are being introduced into the fungicide marketplace. Fluopyram ( $N$-[2-[3chloro-5-(trifluoromethyl)-2-pyridinyl] ethyl]-2 (trifluoromethyl) benzamide) was recently launched by Bayer CropScience Co. and was registered on pistachio in 2012. For resistance management purposes, fluopyram is labeled as products premixed with the demethylation inhibitor (DMI) tebuconazole (trade name: Luna Experience) and the QoI trifloxystrobin (trade name: Luna Sensation). Penthiopyrad ((R,S)-N-[2-(1,3-dimethylbutyl)thien-3-yl]-1methyl-3-trifluoromethyl-1H-yrazole-4-carboxamide) (Mitsui Chemicals), available as Fontelis (DuPont), and fluxapyroxad (3-(difluoromethyl)-1-methyl- $N$-(3',4',5'-trifluorobiphenyl-2-yl)pyrazole-4carboxamide), which is formulated as stand-alone product in $\mathrm{Xe}$ mium (BASF, The Chemical Co.) and in mixture with the active ingredient pyraclostrobin in the premix Merivon (BASF, The Chemical Co.), are two other new SDHIs that are expected to be available for the control of ALB in the near future (most likely in the last quarter of 2013). Because penthiopyrad, fluopyram, and fluxapyroxad are in the same cross-resistance group with boscalid, the rational introductions of these new chemistries into ALB fungicide spray programs would require a good knowledge about the frequencies of boscalid-resistant populations in pistachio orchards and a clear understanding of their cross-resistance patterns with the former SDHI. Because of potential cross resistance, the same mutations in $A a S D H$ genes conferring resistance to boscalid in Alternaria populations would also confer resistance to the new SDHIs and, hence, render them ineffective and useless against ALB in pistachio orchards where widespread resistance to boscalid is prevalent. In a preliminary cross-resistance study, we analyzed the sensitivity profiles to penthiopyrad and fluopyram of a limited number of boscalid-resistant mutants containing mutations in $S D H B$, $S D H C$, and $S D H D$ and wild-type isolates of A. alternata and revealed some discrepancy in cross-resistance patterns between boscalid and fluopyram (3). As expected, A. alternata boscalidresistant isolates carrying $\mathrm{SDH}$-mutations were not affected by penthiopyrad. In contrast, A alternata boscalid-resistant mutants carrying an SDHB-H277Y-substitution were sensitive to fluopyram, whereas another A. alternata boscalid-resistant isolate carrying the H134R substitution in AaSDHC had only a low resistance to fluopyram (3). No information on the sensitivity of A. alternata to fluxapyroxad and on the true baseline sensitivities of this fungus to all the new SDHIs fungicides is available.

The objectives of this study were to (i) establish the baseline sensitivities of A. alternata isolates to fluopyram, penthiopyrad, and fluxapyroxad for a group of isolates collected from pistachio orchards that had never been exposed to any SDHI fungicides; (ii) determine the sensitivities to boscalid, penthiopyrad, fluxapyroxad, and fluopyram for a large number of A. alternata isolates collected from commercial pistachio orchards where boscalid had been used and further investigate cross-resistance patterns between fluopyram, penthiopyrad, fluxapyroxad, and the former SDHI boscalid; and (iii) check which known molecular mechanisms are determining this resistance in resistant phenotypes.

\section{Materials and Methods}

Isolate collections. A collection of 50 single-spore isolates of $A$. alternata, collected during 1999 and 2000 from commercial pistachio orchards with no previous history of exposure to any SDHI fungicide, were used for detecting baseline sensitivity of $A$. alternata to penthiopyrad, fluopyram, and fluxapyroxad. In total, 117 single-spore isolates of A. alternata were collected in 2010 from several commercial pistachio orchards where Pristine had been used. Identification of A. alternata was performed according to a previously established protocol (20). One hundred symptomless buds were collected per orchard just before emerging at the beginning of April. The surface of the buds was sterilized by soaking them in $0.5 \%$ sodium hypochlorite $(10 \%$ commercial bleach solution) for $3 \mathrm{~min}$. Ten buds per plate were then placed on the surface of acidified (25\% [vol/vol] lactic acid at $2.5 \mathrm{ml} \mathrm{liter}^{-1}$ ) potato dextrose agar (APDA; $4 \mathrm{~g}$ of potato infusion, $20 \mathrm{~g}$ of dextrose, and $15 \mathrm{~g}$ of agar per liter) in one $90-\mathrm{mm}$ petri dish. These dishes were incubated at $27^{\circ} \mathrm{C}$ for 6 to 7 days. Per orchard, 10 different Alternaria colonies were selected and a mycelial plug (5 by $5 \mathrm{~mm}$ ) from each colony was transferred to a fresh APDA plate to get a clean isolate without any other pathogen. A part of each colony $\left( \pm 1 \mathrm{~cm}^{2}\right)$ was suspended in $8 \mathrm{ml}$ of sterile water to make single-spore isolations. Each suspension was shaken with a vortex for $10 \mathrm{~s}$ to get the spores released from the agar. Aliquots of 40 and $400 \mu \mathrm{l}$ were spread on a petri dish containing water agar medium $\left(20 \mathrm{~g}\right.$ of agar liter $\left.^{-1}\right)$. The petri dishes were incubated at $30^{\circ} \mathrm{C}$ for $16 \mathrm{~h}$ and then examined with a dissecting microscope. A small piece of agar containing a single germinating spore was cut out from each plate with a sterile surgical blade and transferred to an APDA petri dish.

Determination of sensitivities of $A$. alternata isolates to SDHI fungicides. Sensitivities to penthiopyrad, fluopyram, and fluxapyroxad of baseline isolates and isolates from boscalid-exposed orchards were estimated in mycelial growth tests in YBA agar medium $(19,25)$ containing $10 \mathrm{~g}$ of yeast extract, $10 \mathrm{~g}$ of bacto peptone, $20 \mathrm{~g}$ of sodium acetate, and $15 \mathrm{~g}$ of agar per liter of medium. YBA agar medium was amended with fungicides at concentrations of 0 (control), 0.001, 0.005, 0.01, 0.05, 0.1, 0.5, 1.0, 5.0 , and $10 \mu \mathrm{g} \mathrm{ml}^{-1}$. Mycelial growth for the resistant isolates was further tested at two additional concentrations of fluopyram at 50 and $100 \mu \mathrm{g} \mathrm{ml}^{-1}$. Baseline sensitivity of A. alternata to boscalid was previously established in mycelial growth assays on potato dextrose agar (PDA) medium $(1,4)$ but it was reestablished with the same isolates in YBA medium as described above to allow comparison with the sensitivities to the new SDHIs. Technicalgrade fluopyram (a.i. $97.78 \%$; Bayer CropScience), penthiopyrad (a.i. 99.50\%; DuPont), and commercial products of fluxapyroxad (300 SC; BASF, The Chemical Company) and boscalid (Endura, 70 WG; BASF, The Chemical Company) were dissolved in acetone (fluopyram) or water (fluxapyroxad and boscalid) to obtain stock solutions containing $10 \mathrm{~g}$ a.i. liter $^{-1}$, and serially diluted in acetone or water. Aliquots were then added to autoclaved YBA agar media cooled to $45^{\circ} \mathrm{C}$ to obtain YBA agar media amended with final concentrations of fungicide of 0 (control), 0.001, 0.005, 0.01, 0.05, $0.1,0.5,1.0,5.0,10.0$, or $100.0 \mu \mathrm{g} \mathrm{ml}^{-1}$. The final concentration of acetone in the medium was $1 \%(\mathrm{vol} / \mathrm{vol})$. The effective concentration that inhibits growth by $50 \%\left(\mathrm{EC}_{50}\right)$ value was estimated for each isolate by regressing the probit-transformed relative growth inhibition on the logarithm of fungicide concentration using SAS PROC REG (SAS v. 9.3; SAS Institute). A. alternata isolates were classified into six categories based on in vitro SDHI sensitivity. Isolates with fungicide $\mathrm{EC}_{50}$ values lower than $0.01 \mu \mathrm{g}$ $\mathrm{ml}^{-1}$ were considered highly sensitive to the fungicide, isolates with $\mathrm{EC}_{50}$ values between 0.01 and $1 \mu \mathrm{g} \mathrm{m} \mathrm{m}^{-1}$ were considered sensitive, isolates with $\mathrm{EC}_{50}$ values between 1 and $5 \mu \mathrm{g} \mathrm{ml}^{-1}$ were considered to have a reduced sensitivity, isolates with $\mathrm{EC}_{50}$ values between 5 and $10 \mu \mathrm{g} \mathrm{ml}^{-1}$ were considered to have a low level of resistance, isolates with $\mathrm{EC}_{50}$ values between 10 and $100 \mu \mathrm{g} \mathrm{ml}^{-1}$ were considered to have a moderate level resistance, and isolates with $\mathrm{EC}_{50}$ values greater than $100 \mu \mathrm{g} \mathrm{ml}^{-1}$ were considered to have a high level of resistance. The distributions of the $\mathrm{EC}_{50}$ values for the populations were determined. Associations among baseline sensitivities of each fungicide were evaluated using Pearson correlation analysis (PROC CORR) in SAS (version 9.3). Resistance factors $\left(R_{\mathrm{f}}\right)$ were calculated by dividing the $\mathrm{EC}_{50}$ value for each isolate by the $\mathrm{EC}_{50}$ of the most sensitive isolate for a specific fungicide (lowest $\mathrm{EC}_{50}$ ). 
Genomic DNA isolation, allele-specific polymerase chain reaction, and cleaved amplified polymorphic sequence assays. To elucidate the molecular mechanism of resistance to boscalid, 82 A. alternata isolates (69 isolates highly resistant to boscalid, 1 moderately boscalid-resistant isolate, 1 isolate with reduced sensitivity to boscalid, and 11 boscalid-sensitive isolates) were screened for the presence of known mutations in all three $S D H$ target genes using previously developed polymerase chain reaction (PCR)based assays. Genomic DNA was extracted from mycelium of 10day-old colonies of each A. alternata isolates using the FastDNA Kit (Qbiogene). The manufacturer's protocol was used with small adjustments. The samples were processed three times for $40 \mathrm{~s}$ at speed 5 in the FastPrep instrument. The product was centrifuged for $10 \mathrm{~min}$ at $14,000 \times \mathrm{g}$. Binding matrix $(100 \mu \mathrm{l})$ and salton wash $(400 \mu \mathrm{l})$ was added to the supernatant. After incubation for $10 \mathrm{~min}$, the supernatant was removed and $500 \mu$ l of SEWS-M (salt ethanol wash solution) was added to the pellet. After incubation for $5 \mathrm{~min}$, spinning at $14,000 \times g$, and removing all supernatant, $100 \mu \mathrm{l}$ of DES (DNA elution solution - ultra pure water) to the pellet. This was suspended and incubated in a water bath at $65^{\circ} \mathrm{C}$ for $15 \mathrm{~min}$. The sample was centrifuged for $2 \mathrm{~min}$ at $14,000 \times g$ and the supernatant was transferred to a new tube.

Allele-specific (As)PCR and cleaved amplified polymorphic sequence (CAPS) analyses $(5,6)$ were used to screen for the presence of the point mutations reportedly associated with boscalid resistance. AsPCR developed by Avenot et al. (5) was used to test the presence of the point mutation (C to T) at nucleotide position 996 of the $A a S D H B$ gene which results in an exchange of a histidine at position 277 by a tyrosine (H277Y). A CAPS analysis with the restriction enzyme AciI (5) was used to detect the point mutation from A to $\mathrm{G}$ at position 997 in the $A a S D H B$ gene that caused a change of codon 277 from the histidine residue to arginine (H277R) (5). A CAPS analysis with the restriction enzyme AciI (6) was used to detect the point mutation (A to $\mathrm{G}$ transition) at nucleotide position 490 in the $A a S D H C$ gene which causes a replacement of a histidine residue at codon 134 by an arginine (H134R) (6). A CAPS analysis with the restriction enzyme $X \mathrm{~cm} \mathrm{I}$ (6) was used to detect the point mutation (A to $G$ transition) at nucleotide position 398 in the AaSDHD gene which causes a substitution of a histidine residue at codon 133 by an arginine (H133R) (6). Portions of the AaSDHB, AaSDHC, and AaSDHD genes potentially carrying mutations were PCR amplified using specific primer sets as previously described $(5,6)$, and specific PCR products were directly digested with specific restriction enzymes according to the manufacturer's instructions. Digestion products were subsequently separated by electrophoresis on a $3 \%$ agarose gel in $1 \times$ Tris-acetate-EDTA $(40 \mathrm{mM}$ Tris acetate and $1 \mathrm{mM}$ EDTA) buffer.

\section{Results}

Baseline sensitivities of $A$. alternata to fluopyram, penthiopyrad, and fluxapyroxad. In this study, a mycelial growth assay was used to establish the baseline sensitivities of A. alternata to penthiopyrad, fluopyram, and fluxapyroxad with 50 isolates collected from pistachio orchards without a prior exposure to any SDHI. For comparison, the sensitivity to boscalid for the same isolates was also established. All fungicides (fluopyram, penthiopyrad, fluxapyroxad, and boscalid) were effective in inhibiting the growth of the majority of the A. alternata isolates. Isolate phenotypes and frequency distributions in the unexposed population for these fungicides are summarized in Table 1.

Boscalid sensitivity tests performed on 43 A. alternata isolates showed that the majority $(n=36)$ were sensitive to boscalid with $\mathrm{EC}_{50}$ of 0.011 to $0.650 \mu \mathrm{g} \mathrm{ml}^{-1}$ (mean $\mathrm{EC}_{50}=0.188 \mu \mathrm{g} \mathrm{ml}^{-1}$ ) (Table 1). Four isolates (2-F52, 2-F65, 2-F67, and 2-F69) had reduced sensitivity to boscalid with $\mathrm{EC}_{50}$ of $2.355,4.357,2.363$, and 2.184 $\mu \mathrm{g} \mathrm{ml}^{-1}$, respectively (Table 1). One (2-F59) and two (2-F34 and 3$\mathrm{J} 11)$ baseline isolates had low $\left(\mathrm{EC}_{50}=5.148 \mu \mathrm{g} \mathrm{ml}^{-1}\right)$ and moderate $\left(\mathrm{EC}_{50}\right.$ of 10.26 and $11.601 \mu \mathrm{g} \mathrm{ml}^{-1}$ ) levels of resistance, respectively, to boscalid (Table 1). Fluopyram sensitivity tests performed on 46 A. alternata isolates showed that all of them were sensitive to fluopyram, with $\mathrm{EC}_{50}$ values of 0.014 to $0.957 \mu \mathrm{g} \mathrm{ml}^{-1}$ (mean $\mathrm{EC}_{50}=0.238 \mu \mathrm{g} \mathrm{ml}^{-1}$; Table 1$)$. Penthiopyrad sensitivity tests performed on $43 \mathrm{~A}$. alternata isolates showed that the majority were sensitive to penthiopyrad, with $\mathrm{EC}_{50}$ values of 0.02 to $0.463 \mu \mathrm{g} \mathrm{ml}^{-1}$ (mean $\mathrm{EC}_{50}=0.149 \mu \mathrm{g} \mathrm{ml}^{-1}$; Table 1). Three isolates (2-F78, 2G13, and 3-J06) were highly sensitive to penthiopyrad $\left(\mathrm{EC}_{50}<\right.$ $\left.0.01 \mu \mathrm{g} \mathrm{ml}^{-1}\right)$. One isolate $(2-\mathrm{F} 63)$ had reduced sensitivity to penthiopyrad, with an $\mathrm{EC}_{50}$ value of $2.154 \mu \mathrm{g} \mathrm{ml}^{-1}$ (Table 1). Fluxapyroxad sensitivity tests were performed on 50 A. alternata isolates: 12 isolates were highly sensitive to fluxapyroxad $\left(\mathrm{EC}_{50}<\right.$ $0.01 \mu \mathrm{g} \mathrm{ml}^{-1}$ ) whereas 38 were sensitive, with $\mathrm{EC}_{50}$ values of 0.01 to $0.311 \mu \mathrm{g} \mathrm{ml}^{-1}$ (mean $\mathrm{EC}_{50}=0.058 \mu \mathrm{g} \mathrm{ml}^{-1}$; Table 1).

All the isolates with reduced sensitivity and low and moderate levels of resistance to boscalid remained sensitive to the other three SDHI fungicides. Pearson correlation analysis indicated that there were significant positive baseline sensitivity relationships between boscalid and fluxapyroxad sensitivities $(P=0.0137, r=0.40)$ and fluxapyroxad and penthiopyrad $(P=0.0006, r=0.54)$, and no other relationships were significant. Analysis of 81 sensitive isolates from both baseline and boscalid-exposed populations showed that boscalid had significantly less strong activity against $A$. alternata $(P=0.05)$.

Sensitivity of $A$. alternata isolates from boscalid-exposed pistachio orchards to boscalid and the new SDHIs fluopyram, penthiopyrad, and fluxapyroxad. In this study, $117 \mathrm{~A}$. alternata isolates were obtained from several commercial pistachio orchards that have been exposed to boscalid and tested for their sensitivity to boscalid, penthiopyrad, fluopyram, and fluxapyroxad.

Based on the boscalid sensitivity tests, the 117 A. alternata isolates could be separated into four different phenotypes. In all, $44 \mathrm{~A}$. alternata isolates were sensitive to boscalid, with $\mathrm{EC}_{50}$ values of 0.01 to $0.83 \mu \mathrm{g} \mathrm{ml}^{-1}$ (mean $\mathrm{EC}_{50}=0.251 \mu \mathrm{g} \mathrm{ml}^{-1}$; Table 2). Three isolates (4-A69, 4-B41, and 4-B31) had reduced sensitivity to boscalid, with $\mathrm{EC}_{50}$ of $1.3,3.37$, and $4.01 \mu \mathrm{g} \mathrm{ml}^{-1}$, respectively. One isolate (4-B54) had a moderate level of resistance to boscalid, with an $\mathrm{EC}_{50}$ value of $61.51 \mu \mathrm{g} \mathrm{ml}^{-1}$ (Table 2). Finally, 69 isolates were highly resistant to boscalid, with $\mathrm{EC}_{50}$ values $>100 \mu \mathrm{g} \mathrm{ml}^{-1}$ (Table 2).

Based on the fluopyram sensitivity tests, the 117 A. alternata isolates could be separated into four different phenotypes. Three

Table 1. Values for 50\% effective concentration $\left(\mathrm{EC}_{50}\right)$ of boscalid, fluopyram, penthiopyrad, and fluxapyroxad, and associated phenotypes of Alternaria alternata isolates collected between 1998 and 2003 from California pistachio orchards with no history of succinate dehydrogenase inhibitor usage

\begin{tabular}{|c|c|c|c|c|c|c|c|c|c|c|c|c|}
\hline \multirow[b]{3}{*}{ Class $^{\mathbf{a}}$} & \multicolumn{12}{|c|}{$\mathrm{EC}_{50}$ values $\left(\mu \mathrm{g} \mathrm{ml}^{-1}\right)$ for isolates } \\
\hline & \multicolumn{3}{|c|}{ Boscalid $(n=43)$} & \multicolumn{3}{|c|}{ Fluopyram $(n=46)$} & \multicolumn{3}{|c|}{ Penthiopyrad $(n=43)$} & \multicolumn{3}{|c|}{ Fluxapyroxad $(n=50)$} \\
\hline & Range & Mean & $\mathrm{Fq}(\%)^{\mathrm{b}}$ & Range & Mean & $\mathrm{Fq}(\%)^{\mathrm{b}}$ & Range & Mean & $\mathbf{F q}(\%)^{\mathbf{b}}$ & Range & Mean & $\mathrm{Fq}(\%)^{\mathbf{b}}$ \\
\hline HS & & & & & 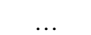 & & $<0.01$ & $<0.01$ & 6.976 & $<0.01$ & $<0.01$ & 24 \\
\hline $\mathrm{S}$ & $0.011-0.650$ & 0.188 & 83.720 & $0.014-0.957$ & 0.238 & 100 & $0.020-0.463$ & 0.149 & 90.697 & $0.010-0.311$ & 0.058 & 76 \\
\hline RS & $2.184-4.357$ & 2.814 & 9.302 & $\ldots$ & $\ldots$ & 0 & 2.154 & 2.154 & 2.325 & $\ldots$ & $\ldots$ & 0 \\
\hline LR & 5.148 & 5.148 & 2.325 & $\ldots$ & $\ldots$ & 0 & $\ldots$ & $\ldots$ & 0 & $\ldots$ & $\ldots$ & 0 \\
\hline MR & $10.260-11.601$ & 10.930 & 4.651 & $\ldots$ & $\ldots$ & 0 & $\ldots$ & $\ldots$ & 0 & $\ldots$ & $\ldots$ & 0 \\
\hline
\end{tabular}

a Phenotype class: $\mathrm{HS}=$ highly sensitive, $\mathrm{S}=$ sensitive, $\mathrm{RS}=$ reduced sensitivity, $\mathrm{LR}=$ low resistance, and MR = moderately resistant.

${ }^{\mathrm{b}}$ Frequency. 
isolates (4-A80, 4-A91, and 4-B17) were highly sensitive to fluopyram $\left(\mathrm{EC}_{50}=0.001 \mu \mathrm{g} \mathrm{ml}^{-1}\right.$; Table 2$)$. In all, $106 \mathrm{~A}$. alternata isolates were sensitive to fluopyram, with $\mathrm{EC}_{50}$ values of 0.01 to $0.91 \mu \mathrm{g} \mathrm{ml}^{-1}$ (mean $\mathrm{EC}_{50}=0.148 \mu \mathrm{g} \mathrm{ml}^{-1}$; Table 2). Seven isolates (4-B16, 4-B18, 4-A42, 4-A57, 4-A81, 4-A24, and 4-A78) had reduced sensitivity to fluopyram, with $\mathrm{EC}_{50}$ values of 1.02 to 2.93 $\mu \mathrm{g} \mathrm{ml}^{-1}$ (mean $\mathrm{EC}_{50}=2.023 \mu \mathrm{g} \mathrm{ml}^{-1}$ ). One isolate (4-B20) had a moderate level of resistance to fluopyram, with an $\mathrm{EC}_{50}$ value of $22.9 \mu \mathrm{g} \mathrm{ml}^{-1}$ (Table 2).
Of 117 A. alternata isolates, 110 were tested for their sensitivity to penthiopyrad. Based on these sensitivity tests, these isolates could be separated into five different phenotypes. In total, $51 \mathrm{~A}$. alternata isolates were sensitive to penthiopyrad, with $\mathrm{EC}_{50}$ values of 0.02 to $0.88 \mu \mathrm{g} \mathrm{ml}^{-1}$ (mean $\mathrm{EC}_{50}=0.224 \mu \mathrm{g} \mathrm{ml}^{-1}$; Table 2) and 13 isolates had reduced sensitivity to penthiopyrad, with $\mathrm{EC}_{50}$ values of 1 to $4.9 \mu \mathrm{g} \mathrm{ml}^{-1}$ (mean $\mathrm{EC}_{50}=3.152 \mu \mathrm{g} \mathrm{ml}^{-1}$; Table 2). In all, 6, 21, and 19 isolates had low $\left(5.1<\mathrm{EC}_{50}<9.3 \mu \mathrm{g} \mathrm{ml}^{-1}\right.$; mean $\left.\mathrm{EC}_{50}=7.632 \mu \mathrm{g} \mathrm{ml}^{-1}\right)$, moderate $\left(10.19<\mathrm{EC}_{50}<83.43 \mu \mathrm{g} \mathrm{ml}^{-1}\right.$;

Table 2. Sensitivity to succinate dehydrogenase (SDH) inhibitor fungicides of Alternaria alternata isolates collected in 2010 from several commercial pistachio orchards with a history of boscalid usage and presence of known amino acid alterations in SDH subunits

\begin{tabular}{|c|c|c|c|c|c|c|c|c|c|c|}
\hline \multirow[b]{2}{*}{ Isolate } & \multirow[b]{2}{*}{ City } & \multirow[b]{2}{*}{ County } & \multicolumn{4}{|c|}{ EC $_{50}$ values $^{\mathrm{a}}$} & \multirow[b]{2}{*}{ Phenotype $^{c}$} & \multicolumn{3}{|c|}{ Amino acid alterations ${ }^{b}$} \\
\hline & & & Bosc & Fluop & Penth & Flux & & SDHB & SDHC & SDHD \\
\hline 4-A66 & Parlier & Fresno & 0.29 & 0.09 & 0.04 & 0.01 & $\mathrm{Bo}^{\mathrm{S}} \mathrm{Fp}^{\mathrm{S}} \mathrm{Pe}^{\mathrm{S}} \mathrm{Fd}^{\mathrm{S}}$ & N.F. & N.F. & N.F. \\
\hline 4-A67 & Parlier & Fresno & 0.21 & 0.03 & 0.54 & 0.11 & $\mathrm{Bo}^{\mathrm{S}} \mathrm{Fp}^{\mathrm{S}} \mathrm{Pe}^{\mathrm{S}} \mathrm{Fd}^{\mathrm{S}}$ & N.I. & N.I. & N.I. \\
\hline 4-A69 & Parlier & Fresno & 1.30 & 0.34 & 0.04 & 0.03 & $\mathrm{Bo}^{\mathrm{RS}} \mathrm{Fp}^{\mathrm{S}} \mathrm{Pe}^{\mathrm{S}} \mathrm{Fd}^{\mathrm{S}}$ & N.I. & N.I. & N.I. \\
\hline 4-A70 & Parlier & Fresno & 0.04 & 0.34 & 0.27 & 0.03 & $\mathrm{Bo}^{\mathrm{S}} \mathrm{Fp}^{\mathrm{S}} \mathrm{Pe}^{\mathrm{S}} \mathrm{Fd}^{\mathrm{S}}$ & N.I. & N.I & N.I. \\
\hline 4-A71 & Parlier & Fresno & 0.09 & 0.40 & 0.20 & 0.23 & $\mathrm{Bo}^{\mathrm{S}} \mathrm{Fp}^{\mathrm{S}} \mathrm{Pe}^{\mathrm{S}} \mathrm{Fd}^{\mathrm{S}}$ & N.I. & N.I & N.I. \\
\hline 4-A73 & Parlier & Fresno & 0.06 & 0.09 & 0.25 & 0.01 & $\mathrm{Bo}^{\mathrm{S} F p} \mathrm{PPe}^{\mathrm{S} F d^{\mathrm{S}}}$ & N.F. & N.F. & N.F. \\
\hline 4-A74 & Parlier & Fresno & 0.01 & 0.02 & 0.06 & 0.001 & $\mathrm{Bo}^{\mathrm{S}} \mathrm{Fp}^{\mathrm{S}} \mathrm{Pe}^{\mathrm{S}} \mathrm{Fd}^{\mathrm{HS}}$ & N.I. & N.I & N.I. \\
\hline 4-A72 & Parlier & Fresno & $>100$ & 0.16 & 0.58 & $>100$ & $\mathrm{Bo}^{\mathrm{HR}} \mathrm{Fp}^{\mathrm{S}} \mathrm{Pe}^{\mathrm{S}} \mathrm{Fd}^{\mathrm{HR}}$ & $\mathrm{H} 277 \mathrm{Y}$ & N.F. & N.F. \\
\hline 4-A95 & Terrabella & Tulare & 0.40 & 0.13 & 0.03 & 0.13 & $\mathrm{Bo}^{\mathrm{S}} \mathrm{Fp}^{\mathrm{S}} \mathrm{Pe}^{\mathrm{S}} \mathrm{Fd}^{\mathrm{S}}$ & N.F. & N.F. & N.F. \\
\hline 4-A96 & Terrabella & Tulare & 0.44 & 0.12 & 0.15 & 0.11 & 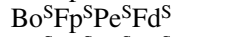 & N.I. & N.I & N.I. \\
\hline 4-A97 & Terrabella & Tulare & 0.30 & 0.03 & 0.03 & 0.16 & $\mathrm{Bo}^{\mathrm{S}} \mathrm{Fp}^{\mathrm{S}} \mathrm{Pe}^{\mathrm{S}} \mathrm{Fd}^{\mathrm{S}}$ & N.I. & N.I & N.I. \\
\hline 4-A98 & Terrabella & Tulare & 0.05 & 0.16 & 0.17 & 0.13 & $\mathrm{Bo}^{\mathrm{S}} \mathrm{Fp}^{\mathrm{S}} \mathrm{Pe}^{\mathrm{S}} \mathrm{Fd}^{\mathrm{S}}$ & N.I. & N.I & N.I. \\
\hline 4-B42 & Terrabella & Tulare & 0.16 & 0.11 & N.D. & 0.20 & $\mathrm{Bo}^{\mathrm{S}} \mathrm{Fp}^{\mathrm{S}} \mathrm{Pe}^{\mathrm{N} \cdot \mathrm{D}} \cdot \mathrm{Fd}^{\mathrm{S}}$ & N.I. & N.I & N.I. \\
\hline 4-B44 & Terrabella & Tulare & 0.25 & 0.22 & 0.07 & 0.09 & $\mathrm{Bo}^{\mathrm{S} F p} \mathrm{~F}^{\mathrm{S}} \mathrm{e}^{\mathrm{S} F d^{\mathrm{S}}}$ & N.F. & N.F. & N.F. \\
\hline 4-B46 & Terrabella & Tulare & 0.14 & 0.01 & 0.02 & 0.02 & $\mathrm{Bo}^{\mathrm{S}} \mathrm{Fp}^{\mathrm{S}} \mathrm{Pe}^{\mathrm{S}} \mathrm{Fd}^{\mathrm{S}}$ & N.I. & N.I & N.I. \\
\hline 4-B47 & Terrabella & Tulare & 0.39 & 0.19 & 0.02 & 0.001 & $\mathrm{Bo}^{\mathrm{S}} \mathrm{Fp}^{\mathrm{S}} \mathrm{Pe}^{\mathrm{S}} \mathrm{Fd}^{\mathrm{HS}}$ & N.I. & N.I & N.I. \\
\hline 4-B41 & Terrabella & Tulare & 3.37 & 0.13 & 0.03 & 0.01 & $\mathrm{Bo}^{\mathrm{RS}} \mathrm{Fp}^{\mathrm{S}} \mathrm{Pe}^{\mathrm{S}} \mathrm{Fd}^{\mathrm{S}}$ & N.I. & N.I. & N.I. \\
\hline 4-B39 & Terrabella & Tulare & 0.56 & 0.02 & 0.05 & 0.10 & $\mathrm{Bo}^{\mathrm{S}} \mathrm{Fp}^{\mathrm{S}} \mathrm{Pe}^{\mathrm{S}} \mathrm{Fd}^{\mathrm{S}}$ & N.I. & N.I & N.I. \\
\hline 4-B43 & Terrabella & Tulare & $>100$ & 0.12 & 13.12 & 22.06 & $\mathrm{Bo}^{\mathrm{HR}} \mathrm{Fp}^{\mathrm{S}} \mathrm{Pe}^{\mathrm{MR}} \mathrm{Fd}^{\mathrm{MR}}$ & $\mathrm{H} 277 \mathrm{Y}$ & N.F. & N.F. \\
\hline 4-B45 & Terrabella & Tulare & $>100$ & 0.91 & 46.67 & 33.84 & $\mathrm{Bo}^{\mathrm{HR}} \mathrm{Fp}^{\mathrm{S}} \mathrm{Pe}^{\mathrm{MR}} \mathrm{Fd}^{\mathrm{MR}}$ & N.F. & H134R & N.F. \\
\hline 4-B32 & Corcoran & Kings & 0.60 & 0.57 & N.D. & 0.01 & $\mathrm{Bo}^{\mathrm{S}} \mathrm{Fp}^{\mathrm{S}} \mathrm{Pe}^{\mathrm{N} \cdot \mathrm{D}} \cdot \mathrm{Fd}^{\mathrm{S}}$ & N.F. & N.F. & N.F. \\
\hline 4-B33 & Corcoran & Kings & 0.30 & 0.17 & 0.82 & 0.14 & $\mathrm{Bo}^{\mathrm{S}} \mathrm{Fp}^{\mathrm{S}} \mathrm{Pe}^{\mathrm{S} F d^{\mathrm{S}}}$ & N.I. & N.I & N.I. \\
\hline 4-B34 & Corcoran & Kings & 0.44 & 0.40 & 0.14 & 0.01 & $\mathrm{Bo}^{\mathrm{S}} \mathrm{Fp}^{\mathrm{S}} \mathrm{Pe}^{\mathrm{S}} \mathrm{Fd}^{\mathrm{S}}$ & N.I. & N.I & N.I. \\
\hline 4-B36 & Corcoran & Kings & 0.06 & 0.24 & 0.22 & 0.01 & $\mathrm{Bo}^{\mathrm{S}} \mathrm{Fp}^{\mathrm{S}} \mathrm{Pe}^{\mathrm{S}} \mathrm{Fd}^{\mathrm{S}}$ & N.I. & N.I & N.I. \\
\hline 4-B31 & Corcoran & Kings & 4.01 & 0.66 & 1.01 & 1.40 & $\mathrm{Bo}^{\mathrm{RS}} \mathrm{Fp}^{\mathrm{S}} \mathrm{Pe}^{\mathrm{RS}} \mathrm{Fd}^{\mathrm{RS}}$ & N.F. & N.F. & N.F. \\
\hline 4-B38 & Corcoran & Kings & $>100$ & 0.27 & $>100$ & $>100$ & $\mathrm{Bo}^{\mathrm{HR}} \mathrm{Fp}^{\mathrm{S}} \mathrm{Pe}^{\mathrm{HR}} \mathrm{Fd}^{\mathrm{HR}}$ & $\mathrm{H} 277 \mathrm{Y}$ & N.F. & N.F. \\
\hline 4-B37 & Corcoran & Kings & 0.80 & 0.03 & 0.43 & 0.01 & $\mathrm{Bo}^{\mathrm{S}} \mathrm{Fp}^{\mathrm{S}} \mathrm{Pe}^{\mathrm{S}} \mathrm{Fd}^{\mathrm{S}}$ & N.F. & H134R & N.F. \\
\hline 4-A58 & Madera & Madera & 0.55 & 0.07 & 0.05 & 0.02 & $\mathrm{Bo}^{\mathrm{S}} \mathrm{Fp}^{\mathrm{S}} \mathrm{Pe}^{\mathrm{S}} \mathrm{Fd}^{\mathrm{S}}$ & N.I. & N.I. & N.I. \\
\hline 4-A48 & Madera & Madera & 0.81 & 0.06 & 0.64 & 0.42 & $\mathrm{Bo}^{\mathrm{S}} \mathrm{Fp}^{\mathrm{S}} \mathrm{Pe}^{\mathrm{S}} \mathrm{Fd}^{\mathrm{S}}$ & N.I. & N.I. & N.I. \\
\hline 4-A50 & Madera & Madera & 0.02 & 0.13 & 0.02 & 0.01 & $\mathrm{Bo}^{\mathrm{S}} \mathrm{Fp}^{\mathrm{S}} \mathrm{Pe}^{\mathrm{S}} \mathrm{Fd}^{\mathrm{S}}$ & N.F. & N.F. & N.F. \\
\hline 4-A63 & Madera & Madera & 0.05 & 0.28 & 0.41 & 0.001 & $\mathrm{Bo}^{\mathrm{S}} \mathrm{Fp}^{\mathrm{S}} \mathrm{Pe}^{\mathrm{S}} \mathrm{Fd}^{\mathrm{HS}}$ & N.I. & N.I. & N.I. \\
\hline 4-A65 & Madera & Madera & 0.39 & 0.50 & 0.24 & 0.02 & $\mathrm{Bo}^{\mathrm{S}} \mathrm{Fp}^{\mathrm{S}} \mathrm{Pe}^{\mathrm{S}} \mathrm{Fd}^{\mathrm{S}}$ & N.I. & N.I. & N.I. \\
\hline 4-A43 & Madera & Madera & $>100$ & 0.01 & 0.13 & 0.04 & $\mathrm{Bo}^{\mathrm{HR}} \mathrm{Fp}^{\mathrm{S}} \mathrm{Pe}^{\mathrm{S}} \mathrm{Fd}^{\mathrm{S}}$ & N.F. & N.F. & N.F. \\
\hline 4-A38 & Madera & Madera & $>100$ & 0.01 & $>100$ & N.D. & $\mathrm{Bo}^{\mathrm{HR}} \mathrm{Fp}^{\mathrm{S}} \mathrm{Pe}^{\mathrm{HR}} \mathrm{Fd}^{\mathrm{N} . \mathrm{D} .}$ & N.F. & N.F. & N.F. \\
\hline 4-A51 & Madera & Madera & $>100$ & 0.04 & 45.88 & 0.09 & $\mathrm{Bo}^{\mathrm{HR}} \mathrm{Fp}^{\mathrm{S}} \mathrm{Pe}^{\mathrm{MR}} \mathrm{Fd}^{\mathrm{S}}$ & $\mathrm{H} 277 \mathrm{Y}$ & N.F. & N.F. \\
\hline 4-A52 & Madera & Madera & $>100$ & 0.01 & 44.87 & 4.27 & $\mathrm{Bo}^{\mathrm{HR}} \mathrm{Fp}^{\mathrm{S}} \mathrm{Pe}^{\mathrm{MR}} \mathrm{Fd}^{\mathrm{RS}}$ & $\mathrm{H} 277 \mathrm{Y}$ & N.F. & N.F. \\
\hline $4-A 53$ & Madera & Madera & $>100$ & 0.05 & 17.95 & 0.13 & $\mathrm{Bo}^{\mathrm{HR}} \mathrm{Fp}^{\mathrm{S}} \mathrm{Pe}^{\mathrm{MR}} \mathrm{Fd}^{\mathrm{S}}$ & $\mathrm{H} 277 \mathrm{Y}$ & N.F. & N.F. \\
\hline 4-A54 & Madera & Madera & $>100$ & 0.30 & 36.38 & $>100$ & $\mathrm{Bo}^{\mathrm{HR}} \mathrm{Fp}^{\mathrm{S}} \mathrm{Pe}^{\mathrm{MR}} \mathrm{Fd}^{\mathrm{HR}}$ & $\mathrm{H} 277 \mathrm{Y}$ & N.F. & N.F. \\
\hline 4-A59 & Madera & Madera & $>100$ & 0.02 & 4.00 & 0.10 & $\mathrm{Bo}^{\mathrm{HR}} \mathrm{Fp}^{\mathrm{S}} \mathrm{Pe}^{\mathrm{RS}} \mathrm{Fd}^{\mathrm{S}}$ & $\mathrm{H} 277 \mathrm{Y}$ & N.F. & N.F. \\
\hline 4-A60 & Madera & Madera & $>100$ & 0.31 & 0.18 & 0.10 & $\mathrm{Bo}^{\mathrm{HR}} \mathrm{Fp}^{\mathrm{S}} \mathrm{Pe}^{\mathrm{S}} \mathrm{Fd}^{\mathrm{S}}$ & $\mathrm{H} 277 \mathrm{Y}$ & N.F. & N.F. \\
\hline 4-A61 & Madera & Madera & $>100$ & 0.01 & $>100$ & 0.20 & $\mathrm{Bo}^{\mathrm{HR}} \mathrm{Fp}^{\mathrm{S}} \mathrm{Pe}^{\mathrm{HR}} \mathrm{Fd}^{\mathrm{S}}$ & $\mathrm{H} 277 \mathrm{Y}$ & N.F. & N.F. \\
\hline 4-A62 & Madera & Madera & $>100$ & 0.02 & 52.15 & $>100$ & $\mathrm{Bo}^{\mathrm{HR}} \mathrm{Fp}^{\mathrm{S}} \mathrm{Pe}^{\mathrm{MR}} \mathrm{Fd}^{\mathrm{HR}}$ & $\mathrm{H} 277 \mathrm{Y}$ & N.F. & N.F. \\
\hline 4-A64 & Madera & Madera & $>100$ & 0.21 & 10.46 & 0.45 & $\mathrm{Bo}^{\mathrm{HR}} \mathrm{Fp}^{\mathrm{S}} \mathrm{Pe}^{\mathrm{MR}} \mathrm{Fd}^{\mathrm{S}}$ & $\mathrm{H} 277 \mathrm{Y}$ & N.F. & N.F. \\
\hline 4-A34 & Madera & Madera & $>100$ & 0.06 & N.D. & 0.06 & $\mathrm{Bo}^{\mathrm{HR}} \mathrm{Fp}^{\mathrm{S}} \mathrm{Pe}^{\mathrm{N} \cdot \mathrm{D}} \cdot \mathrm{Fd}^{\mathrm{S}}$ & $\mathrm{H} 277 \mathrm{Y}$ & N.F. & N.F. \\
\hline 4-A35 & Madera & Madera & $>100$ & 0.01 & $>100$ & 1.01 & $\mathrm{Bo}^{\mathrm{HR}} \mathrm{Fp}^{\mathrm{S}} \mathrm{Pe}^{\mathrm{HR}} \mathrm{Fd}^{\mathrm{RS}}$ & $\mathrm{H} 277 \mathrm{Y}$ & N.F. & N.F. \\
\hline 4-A36 & Madera & Madera & $>100$ & 0.01 & 9.30 & 3.26 & $\mathrm{Bo}^{\mathrm{HR}} \mathrm{Fp}^{\mathrm{S}} \mathrm{Pe}^{\mathrm{LR}} \mathrm{Fd}^{\mathrm{RS}}$ & $\mathrm{H} 277 \mathrm{Y}$ & N.F. & N.F. \\
\hline 4-A39 & Madera & Madera & $>100$ & 0.02 & 11.94 & 0.89 & $\mathrm{Bo}^{\mathrm{HR}} \mathrm{Fp}^{\mathrm{S}} \mathrm{Pe}^{\mathrm{MR}} \mathrm{Fd}^{\mathrm{S}}$ & $\mathrm{H} 277 \mathrm{Y}$ & N.F. & N.F. \\
\hline 4-A40 & Madera & Madera & $>100$ & 0.02 & 8.85 & 5.80 & $\mathrm{Bo}^{\mathrm{HR}} \mathrm{Fp}^{\mathrm{S}} \mathrm{Pe}^{\mathrm{LR}} \mathrm{Fd}^{\mathrm{LR}}$ & $\mathrm{H} 277 \mathrm{Y}$ & N.F. & N.F. \\
\hline 4-A41 & Madera & Madera & $>100$ & 0.01 & 5.10 & 0.24 & $\mathrm{Bo}^{\mathrm{HR}} \mathrm{Fp}^{\mathrm{S}} \mathrm{Pe}^{\mathrm{LR}} \mathrm{Fd}^{\mathrm{S}}$ & $\mathrm{H} 277 \mathrm{Y}$ & N.F. & N.F. \\
\hline 4-A44 & Madera & Madera & $>100$ & 0.01 & 1.76 & 0.31 & $\mathrm{Bo}^{\mathrm{HR}} \mathrm{Fp}^{\mathrm{S}} \mathrm{Pe}^{\mathrm{RS}} \mathrm{Fd}^{\mathrm{S}}$ & $\mathrm{H} 277 \mathrm{Y}$ & N.F. & N.F. \\
\hline 4-A45 & Madera & Madera & $>100$ & 0.01 & 0.45 & 0.20 & $\mathrm{Bo}^{\mathrm{HR}} \mathrm{Fp}^{\mathrm{S}} \mathrm{Pe}^{\mathrm{S}} \mathrm{Fd}^{\mathrm{S}}$ & $\mathrm{H} 277 \mathrm{Y}$ & N.F. & N.F. \\
\hline 4-A46 & Madera & Madera & $>100$ & 0.03 & N.D. & 1.74 & $\mathrm{Bo}^{\mathrm{HR}} \mathrm{Fp}^{\mathrm{S}} \mathrm{Pe}^{\mathrm{N} \cdot \mathrm{D}} \cdot \mathrm{Fd}^{\mathrm{RS}}$ & $\mathrm{H} 277 \mathrm{Y}$ & N.F. & N.F. \\
\hline 4-A47 & Madera & Madera & $>100$ & 0.01 & 0.65 & 1.79 & $\mathrm{Bo}^{\mathrm{HR}} \mathrm{Fp}^{\mathrm{S}} \mathrm{Pe}^{\mathrm{S}} \mathrm{Fd}^{\mathrm{RS}}$ & $\mathrm{H} 277 \mathrm{Y}$ & N.F. & N.F. \\
\hline 4-A37 & Madera & Madera & $>100$ & 0.35 & $>100$ & 69.69 & $\mathrm{Bo}^{\mathrm{HR}} \mathrm{Fp}^{\mathrm{S}} \mathrm{Pe}^{\mathrm{HR}} \mathrm{Fd}^{\mathrm{MR}}$ & N.F. & H134R & N.F. \\
\hline $4-\mathrm{A} 42$ & Madera & Madera & $>100$ & 1.89 & $>100$ & $>100$ & $\mathrm{Bo}^{\mathrm{HR}} \mathrm{Fp}^{\mathrm{RS}} \mathrm{Pe}^{\mathrm{HR}} \mathrm{Fd}^{\mathrm{HR}}$ & N.F. & H134R & N.F. \\
\hline & & & & & & & & & continued & ext page) \\
\hline
\end{tabular}

${ }^{\mathrm{a}} \mathrm{EC}_{50}=50 \%$ effective concentration of boscalid (Bosc), fluopyram (Fluop), penthiopyrad (Penth), and fluxapyroxad (Flux); N.D. = not determined.

${ }^{\mathrm{b}}$ Occurrence of known mutations in $\mathrm{A} a \mathrm{SDH}$ genes of some mutants was investigated using previously developed allele-specific polymerase chain reaction and cleaved amplified polymorphic sequence assays. N.F. $=$ no previous known mutation caused this phenotype of the isolate and N.I. $=$ not investigated.

${ }^{c} \mathrm{Bo}=$ boscalid, $\mathrm{Fp}=$ fluopyram, $\mathrm{Pe}=$ penthiopyrad, $\mathrm{Fd}=$ fluxapyroxad, $\mathrm{HS}=$ highly sensitive, $\mathrm{S}=$ sensitive, $\mathrm{RS}=$ reduced sensitivity, $\mathrm{LR}=$ low resistance, $\mathrm{MR}=$ moderately resistant, $\mathrm{HR}=$ highly resistant. 
mean $\left.\mathrm{EC}_{50}=38.083 \mu \mathrm{g} \mathrm{ml}^{-1}\right)$, and high $\left(\mathrm{EC}_{50}>100 \mu \mathrm{g} \mathrm{ml}^{-1}\right)$ levels of resistance to penthiopyrad, respectively (Table 2 ).

Of 117 A. alternata isolates, 113 were tested for their sensitivity to fluxapyroxad. Based on these sensitivity tests, these isolates could be separated into six different phenotypes. In total, 10 isolates were highly sensitive to fluxapyroxad $\left(\mathrm{EC}_{50}=0.001\right.$ $\mu \mathrm{g} \mathrm{ml}{ }^{-1}$; Table 2); 63 A. alternata isolates were sensitive to fluxapyroxad, with $\mathrm{EC}_{50}$ values of 0.01 to $0.97 \mu \mathrm{g} \mathrm{ml}^{-1}$ (mean $\mathrm{EC}_{50}=0.147 \mu \mathrm{g} \mathrm{ml} \mathrm{g}^{-1}$; Table 2); and 15 isolates had reduced sensitivity to fluxapyroxad, with $\mathrm{EC}_{50}$ values of 1.01 to $4.98 \mu \mathrm{g}$ $\mathrm{ml}^{-1}$ (mean $\mathrm{EC}_{50}=2.383 \mu \mathrm{g} \mathrm{ml}^{-1}$ ). Finally, 2 (4-A24 and 4-A40), 11 , and 12 isolates had low $\left(\mathrm{EC}_{50}\right.$ of 5.53 and $\left.5.8 \mu \mathrm{g} \mathrm{ml}^{-1}\right)$, moderate $\left(10.19<\mathrm{EC}_{50}<91.07 \mu \mathrm{g} \mathrm{ml}^{-1}\right.$; mean $\mathrm{EC}_{50}=30.747 \mu \mathrm{g}$ $\mathrm{ml}^{-1}$ ), and high $\left(\mathrm{EC}_{50}>100 \mu \mathrm{g} \mathrm{ml}^{-1}\right)$ levels of resistance to fluxapyroxad, respectively (Table 2 ).

Pearson correlation analysis with all the isolates tested indicated that there were significant positive sensitivity-resistance relationships between boscalid and penthiopyrad $(P<0.0001, r=0.53)$,

Table 2. (continued from preceding page)

\begin{tabular}{|c|c|c|c|c|c|c|c|c|c|c|}
\hline \multirow[b]{2}{*}{ Isolate } & \multirow[b]{2}{*}{ City } & \multirow[b]{2}{*}{ County } & \multicolumn{4}{|c|}{ EC $_{50}$ values $^{\mathrm{a}}$} & \multirow[b]{2}{*}{ Phenotype $^{c}$} & \multicolumn{3}{|c|}{ Amino acid alterations } \\
\hline & & & Bosc & Fluop & Penth & Flux & & SDHB & SDHC & SDHD \\
\hline 4-A49 & Madera & Madera & $>100$ & 0.02 & 2.24 & 2.63 & $\mathrm{Bo}^{\mathrm{HR}} \mathrm{Fp}^{\mathrm{S}} \mathrm{Pe}^{\mathrm{RS}} \mathrm{Fd}^{\mathrm{RS}}$ & N.F. & H134R & N.F. \\
\hline 4-A55 & Madera & Madera & $>100$ & 0.16 & 12.97 & $>100$ & $\mathrm{Bo}^{\mathrm{HR}} \mathrm{Fp}^{\mathrm{S}} \mathrm{Pe}^{\mathrm{MR}} \mathrm{Fd}^{\mathrm{HR}}$ & N.F. & H134R & N.F. \\
\hline 4-A56 & Madera & Madera & $>100$ & 0.29 & 7.64 & N.D. & $\mathrm{Bo}^{\mathrm{HR}} \mathrm{Fp}^{\mathrm{S}} \mathrm{Pe}^{\mathrm{LR}} \mathrm{Fd}^{\mathrm{N} . \mathrm{D}}$. & N.F. & H134R & N.F. \\
\hline 4-A57 & Madera & Madera & $>100$ & 2.05 & $>100$ & 16.68 & $\mathrm{Bo}^{\mathrm{HR}} \mathrm{Fp}^{\mathrm{RS}} \mathrm{Pe}^{\mathrm{HR}} \mathrm{Fd}^{\mathrm{MR}}$ & N.F. & H134R & N.F. \\
\hline 4-A15 & Hanford & Kings & 0.01 & 0.02 & 0.27 & 0.001 & $\mathrm{Bo}^{\mathrm{S}} \mathrm{Fp}^{\mathrm{S}} \mathrm{Pe}^{\mathrm{S}} \mathrm{Fd}^{\mathrm{HS}}$ & N.I. & N.I. & N.I. \\
\hline 4-A17 & Hanford & Kings & 0.18 & 0.05 & 0.19 & 0.001 & $\mathrm{Bo}^{\mathrm{S}} \mathrm{Fp}^{\mathrm{S}} \mathrm{Pe}^{\mathrm{S}} \mathrm{Fd}^{\mathrm{HS}}$ & N.F. & N.F. & N.F. \\
\hline 4-A18 & Hanford & Kings & 0.04 & 0.07 & 0.25 & 0.001 & $\mathrm{Bo}^{\mathrm{S}} \mathrm{Fp}^{\mathrm{S}} \mathrm{Pe}^{\mathrm{S}} \mathrm{Fd}^{\mathrm{HS}}$ & N.I. & N.I. & N.I. \\
\hline 4-A87 & Hanford & Kings & 0.09 & 0.06 & 0.17 & 0.01 & $\mathrm{Bo}^{\mathrm{S}} \mathrm{Fp}^{\mathrm{S}} \mathrm{Pe}^{\mathrm{S}} \mathrm{Fd}^{\mathrm{S}}$ & N.I. & N.I. & N.I. \\
\hline $4-\mathrm{A} 88$ & Hanford & Kings & 0.12 & 0.11 & 0.04 & 0.08 & $\mathrm{Bo}^{\mathrm{S}} \mathrm{Fp}^{\mathrm{S}} \mathrm{Pe}^{\mathrm{S}} \mathrm{Fd}^{\mathrm{S}}$ & N.I. & N.I. & N.I. \\
\hline $4-A 89$ & Hanford & Kings & 0.10 & 0.02 & 0.04 & 0.02 & $\mathrm{Bo}^{\mathrm{S}} \mathrm{Fp}^{\mathrm{S}} \mathrm{Pe}^{\mathrm{S}} \mathrm{Fd}^{\mathrm{S}}$ & N.I. & N.I. & N.I. \\
\hline 4-A90 & Hanford & Kings & 0.06 & 0.12 & 0.11 & 0.001 & $\mathrm{Bo}^{\mathrm{S}} \mathrm{Fp}^{\mathrm{S}} \mathrm{Pe}^{\mathrm{S}} \mathrm{Fd}^{\mathrm{HS}}$ & N.F. & N.F. & N.F. \\
\hline 4-A91 & Hanford & Kings & 0.11 & 0.001 & 0.20 & 0.01 & $\mathrm{Bo}^{\mathrm{S}} \mathrm{Fp}^{\mathrm{HS}} \mathrm{Pe}^{\mathrm{S}} \mathrm{Fd}^{\mathrm{S}}$ & N.I. & N.I. & N.I. \\
\hline 4-A16 & Hanford & Kings & $>100$ & 0.02 & 7.62 & 0.02 & $\mathrm{Bo}^{\mathrm{HR}} \mathrm{Fp}^{\mathrm{S}} \mathrm{Pe}^{\mathrm{LR}} \mathrm{Fd}^{\mathrm{S}}$ & $\mathrm{H} 277 \mathrm{Y}$ & N.F. & N.F. \\
\hline 4-A19 & Hanford & Kings & $>100$ & 0.17 & $>100$ & 10.64 & $\mathrm{Bo}^{\mathrm{HR}} \mathrm{Fp}^{\mathrm{S}} \mathrm{Pe}^{\mathrm{HR}} \mathrm{Fd}^{\mathrm{MR}}$ & $\mathrm{H} 277 \mathrm{Y}$ & N.F. & N.F. \\
\hline 4-A21 & Hanford & Kings & $>100$ & 0.04 & 30.79 & 10.19 & $\mathrm{Bo}^{\mathrm{HR}} \mathrm{Fp}^{\mathrm{S}} \mathrm{Pe}^{\mathrm{MR}} \mathrm{Fd}^{\mathrm{MR}}$ & $\mathrm{H} 277 \mathrm{Y}$ & N.F. & N.F. \\
\hline 4-A22 & Hanford & Kings & $>100$ & 0.02 & 1.00 & 0.27 & $\mathrm{Bo}^{\mathrm{HR}} \mathrm{Fp}^{\mathrm{S}} \mathrm{Pe}^{\mathrm{RS}} \mathrm{Fd}^{\mathrm{S}}$ & $\mathrm{H} 277 \mathrm{Y}$ & N.F. & N.F. \\
\hline $4-\mathrm{A} 23$ & Hanford & Kings & $>100$ & 0.27 & $>100$ & 3.25 & $\mathrm{Bo}^{\mathrm{HR}} \mathrm{Fp}^{\mathrm{S}} \mathrm{Pe}^{\mathrm{HR}} \mathrm{Fd}^{\mathrm{RS}}$ & $\mathrm{H} 277 \mathrm{Y}$ & N.F. & N.F. \\
\hline $4-\mathrm{A} 24$ & Hanford & Kings & $>100$ & 2.51 & 83.22 & 5.53 & $\mathrm{Bo}^{\mathrm{HR}} \mathrm{Fp}^{\mathrm{RS}} \mathrm{Pe}^{\mathrm{MR}} \mathrm{Fd}^{\mathrm{LR}}$ & N.F. & H134R & N.F. \\
\hline 4-A93 & Shafter & Kern & 0.06 & 0.01 & 0.10 & 0.001 & $\mathrm{Bo}^{\mathrm{S}} \mathrm{Fp}^{\mathrm{S}} \mathrm{Pe}^{\mathrm{S}} \mathrm{Fd}^{\mathrm{HS}}$ & N.I. & N.I. & N.I. \\
\hline 4-A94 & Shafter & Kern & 0.16 & 0.01 & 0.02 & 0.001 & $\mathrm{Bo}^{\mathrm{S}} \mathrm{Fp}^{\mathrm{S}} \mathrm{Pe}^{\mathrm{S}} \mathrm{Fd}^{\mathrm{HS}}$ & N.I. & N.I. & N.I. \\
\hline 4-A83 & Shafter & Kern & 0.05 & 0.21 & 2.96 & 0.06 & $\mathrm{Bo}^{\mathrm{S}} \mathrm{Fp}^{\mathrm{S}} \mathrm{Pe}^{\mathrm{RS}} \mathrm{Fd}^{\mathrm{S}}$ & N.I. & N.I. & N.I. \\
\hline 4-A100 & Shafter & Kern & 0.18 & 0.13 & 0.47 & 0.13 & $\mathrm{Bo}^{\mathrm{S}} \mathrm{Fp}^{\mathrm{S}} \mathrm{Pe}^{\mathrm{S}} \mathrm{Fd}^{\mathrm{S}}$ & N.I. & N.I. & N.I. \\
\hline 4-B 10 & Shafter & Kern & 0.71 & 0.05 & 0.39 & 0.01 & $\mathrm{Bo}^{S} \mathrm{Fp}^{\mathrm{S}} \mathrm{Pe}^{\mathrm{S} F d^{S}}$ & N.I. & N.I. & N.I. \\
\hline 4-B14 & Shafter & Kern & 0.06 & 0.03 & 0.06 & 0.74 & $\mathrm{Bo}^{\mathrm{S}} \mathrm{Fp}^{\mathrm{S}} \mathrm{Pe}^{\mathrm{S}} \mathrm{Fd}^{\mathrm{S}}$ & N.I. & N.I. & N.I. \\
\hline 4-B15 & Shafter & Kern & 0.73 & 0.51 & 0.29 & 0.01 & $\mathrm{Bo}^{\mathrm{S}} \mathrm{Fp}^{\mathrm{S}} \mathrm{Pe}^{\mathrm{S}} \mathrm{Fd}^{\mathrm{S}}$ & N.I. & N.I. & N.I. \\
\hline 4-B16 & Shafter & Kern & 0.02 & 1.02 & 0.31 & 0.02 & $\mathrm{Bo}^{\mathrm{S}} \mathrm{Fp}^{\mathrm{RS}} \mathrm{Pe}^{\mathrm{S}} \mathrm{Fd}^{\mathrm{S}}$ & N.I. & N.I. & N.I. \\
\hline 4-B19 & Shafter & Kern & $>100$ & 0.10 & N.D. & 0.02 & 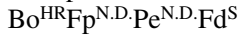 & $\mathrm{H} 277 \mathrm{R}$ & N.F. & N.F. \\
\hline 4-B09 & Shafter & Kern & 0.83 & 0.30 & 0.03 & 0.09 & $\mathrm{Bo}^{\mathrm{S}} \mathrm{Fp}^{\mathrm{S}} \mathrm{Pe}^{\mathrm{S}} \mathrm{Fd}^{\mathrm{S}}$ & N.I. & N.I. & N.I. \\
\hline 4-A84 & Shafter & Kern & $>100$ & 0.47 & $>100$ & 0.01 & $\mathrm{Bo}^{\mathrm{HR}} \mathrm{Fp}^{\mathrm{S}} \mathrm{Pe}^{\mathrm{HR}} \mathrm{Fd}^{\mathrm{S}}$ & N.F. & N.F. & N.F. \\
\hline $4-\mathrm{A} 82$ & Shafter & Kern & $>100$ & 0.20 & $>100$ & 0.10 & $\mathrm{Bo}^{\mathrm{HR}} \mathrm{Fp}^{\mathrm{S}} \mathrm{Pe}^{\mathrm{HR}} \mathrm{Fd}^{\mathrm{S}}$ & $\mathrm{H} 277 \mathrm{Y}$ & N.F. & N.F. \\
\hline 4-B11 & Shafter & Kern & $>100$ & 0.12 & 25.21 & 0.07 & $\mathrm{Bo}^{\mathrm{HR}} \mathrm{Fp}^{\mathrm{S}} \mathrm{Pe}^{\mathrm{MR}} \mathrm{Fd}^{\mathrm{S}}$ & $\mathrm{H} 277 \mathrm{Y}$ & N.F. & N.F. \\
\hline 4-B21 & Shafter & Kern & $>100$ & 0.02 & 18.38 & $>100$ & $\mathrm{Bo}^{\mathrm{HR}} \mathrm{Fp}^{\mathrm{S}} \mathrm{Pe}^{\mathrm{MR}} \mathrm{Fd}^{\mathrm{HR}}$ & $\mathrm{H} 277 \mathrm{Y}$ & N.F. & N.F. \\
\hline 4-B17 & Shafter & Kern & $>100$ & 0.001 & 0.04 & N.D. & $\mathrm{Bo}^{\mathrm{HR}} \mathrm{Fp}^{\mathrm{MS}} \mathrm{Pe}^{\mathrm{S}} \mathrm{Fd}^{\text {N.D. }}$ & $\mathrm{H} 277 \mathrm{Y}$ & N.F. & N.F. \\
\hline 4-A76 & Shafter & Kern & $>100$ & 0.13 & $>100$ & 23.86 & $\mathrm{Bo}^{\mathrm{HR}} \mathrm{Fp}^{\mathrm{S}} \mathrm{Pe}^{\mathrm{HR}} \mathrm{Fd}^{\mathrm{MR}}$ & $\mathrm{H} 277 \mathrm{Y}$ & N.F. & N.F. \\
\hline 4-A77 & Shafter & Kern & $>100$ & 0.01 & N.D. & 19.78 & $\mathrm{Bo}^{\mathrm{HR}} \mathrm{Fp}^{\mathrm{S}} \mathrm{Pe}^{\mathrm{N} \cdot \mathrm{D}} \cdot \mathrm{Fd}^{\mathrm{MR}}$ & $\mathrm{H} 277 \mathrm{Y}$ & N.F. & N.F. \\
\hline 4-A80 & Shafter & Kern & $>100$ & 0.001 & $>100$ & N.D. & $\mathrm{Bo}^{\mathrm{HR}} \mathrm{Fp}^{\mathrm{HS}} \mathrm{Pe}^{\mathrm{HR}} \mathrm{Fd}^{\mathrm{N} . \mathrm{D}}$. & $\mathrm{H} 277 \mathrm{Y}$ & N.F. & N.F. \\
\hline 4-A92 & Shafter & Kern & $>100$ & 0.01 & 47.57 & $>100$ & $\mathrm{Bo}^{\mathrm{HR}} \mathrm{Fp}^{\mathrm{S}} \mathrm{Pe}^{\mathrm{MR}} \mathrm{Fd}^{\mathrm{HR}}$ & $\mathrm{H} 277 \mathrm{Y}$ & N.F. & N.F. \\
\hline 4-B13 & Shafter & Kern & $>100$ & 0.44 & $>100$ & 1.29 & $\mathrm{Bo}^{\mathrm{HR}} \mathrm{Fp}^{\mathrm{S}} \mathrm{Pe}^{\mathrm{HR}} \mathrm{Fd}^{\mathrm{RS}}$ & $\mathrm{H} 277 \mathrm{Y}$ & N.F. & N.F. \\
\hline 4-B18 & Shafter & Kern & $>100$ & 1.52 & $>100$ & 0.001 & $\mathrm{Bo}^{\mathrm{HR}} \mathrm{Fp}^{\mathrm{RS}} \mathrm{Pe}^{\mathrm{HR}} \mathrm{Fd}^{\mathrm{HS}}$ & H134R & N.F. & N.F. \\
\hline 4-B12 & Shafter & Kern & $>100$ & 0.12 & 0.88 & 0.06 & $\mathrm{Bo}^{\mathrm{HR}} \mathrm{Fp}^{\mathrm{SP}} \mathrm{Pe}^{\mathrm{S}} \mathrm{Fd}^{\mathrm{S}}$ & H277R & N.F. & N.F. \\
\hline 4-A81 & Shafter & Kern & $>100$ & 2.24 & 76.64 & 2.12 & $\mathrm{Bo}^{\mathrm{HR}} \mathrm{Fp}^{\mathrm{RS}} \mathrm{Pe}^{\mathrm{MR}} \mathrm{Fd}^{\mathrm{RS}}$ & N.F. & H134R & N.F. \\
\hline 4-A79 & Shafter & Kern & 0.09 & 0.05 & 0.22 & 0.16 & $\mathrm{Bo}^{\mathrm{S}} \mathrm{Fp}^{\mathrm{S}} \mathrm{Pe}^{\mathrm{S}} \mathrm{Fd}^{\mathrm{S}}$ & N.F. & H134R & N.F. \\
\hline $4-\mathrm{B} 20$ & Shafter & Kern & $>100$ & 22.90 & 83.43 & $>100$ & $\mathrm{Bo}^{\mathrm{HR}} \mathrm{Fp}^{\mathrm{MR}} \mathrm{Pe}^{\mathrm{MR}} \mathrm{Fd}^{\mathrm{HR}}$ & N.F. & H134R & N.F. \\
\hline 4-A78 & Shafter & Kern & $>100$ & 2.93 & 4.90 & $>100$ & $\mathrm{Bo}^{\mathrm{HR}} \mathrm{Fp}^{\mathrm{RS}} \mathrm{Pe}^{\mathrm{RS}} \mathrm{Fd}^{\mathrm{HR}}$ & N.F. & N.F. & H133R \\
\hline $4-\mathrm{A} 30$ & Unknown & & $>100$ & 0.02 & 7.28 & 0.97 & $\mathrm{Bo}^{\mathrm{HR}} \mathrm{Fp}^{\mathrm{S}} \mathrm{Pe}^{\mathrm{LR}} \mathrm{Fd}^{\mathrm{S}}$ & $\mathrm{H} 277 \mathrm{Y}$ & N.F. & N.F. \\
\hline $4-B 50$ & Unknown & & 0.02 & 0.17 & 0.09 & 0.01 & $\mathrm{Bo}^{\mathrm{S}} \mathrm{Fp}^{\mathrm{S}} \mathrm{Pe}^{\mathrm{S}} \mathrm{Fd}^{\mathrm{S}}$ & N.F. & N.F. & N.F. \\
\hline 4-A85 & Unknown & & $>100$ & 0.02 & 10.19 & 0.13 & $\mathrm{Bo}^{\mathrm{HR}} \mathrm{Fp}^{\mathrm{S}} \mathrm{Pe}^{\mathrm{MR}} \mathrm{Fd}^{\mathrm{S}}$ & $\mathrm{H} 277 \mathrm{Y}$ & N.F. & N.F. \\
\hline 4-A86 & Unknown & & $>100$ & 0.09 & 2.67 & 14.98 & $\mathrm{Bo}^{\mathrm{HR}} \mathrm{Fp}^{\mathrm{S}} \mathrm{Pe}^{\mathrm{RS}} \mathrm{Fd}^{\mathrm{MR}}$ & $\mathrm{H} 277 \mathrm{Y}$ & N.F. & N.F. \\
\hline 4-A31 & Unknown & & $>100$ & 0.12 & 3.20 & 1.21 & $\mathrm{Bo}^{\mathrm{HR}} \mathrm{Fp}^{\mathrm{S}} \mathrm{Pe}^{\mathrm{RS}} \mathrm{Fd}^{\mathrm{S}}$ & $\mathrm{H} 277 \mathrm{Y}$ & N.F. & N.F. \\
\hline 4-A32 & Unknown & & $>100$ & 0.05 & 0.30 & 0.50 & $\mathrm{Bo}^{\mathrm{HR}} \mathrm{Fp}^{\mathrm{S}} \mathrm{Pe}^{\mathrm{S}} \mathrm{Fd}^{\mathrm{S}}$ & $\mathrm{H} 277 \mathrm{Y}$ & N.F. & N.F. \\
\hline 4-A29 & Unknown & & $>100$ & 0.07 & $>100$ & 0.14 & $\mathrm{Bo}^{\mathrm{HR}} \mathrm{Fp}^{\mathrm{S}} \mathrm{Pe}^{\mathrm{S}} \mathrm{Fd}^{\mathrm{S}}$ & $\mathrm{H} 277 \mathrm{Y}$ & N.F. & N.F. \\
\hline 4-B49 & Unknown & & $>100$ & 0.48 & $>100$ & 25.43 & $\mathrm{Bo}^{\mathrm{HR}} \mathrm{Fp}^{\mathrm{S}} \mathrm{Pe}^{\mathrm{HR}} \mathrm{Fd}^{\mathrm{MR}}$ & $\mathrm{H} 277 \mathrm{Y}$ & N.F. & N.F. \\
\hline 4-B54 & Unknown & & 61.51 & 0.08 & 3.26 & 0.36 & $\mathrm{Bo}^{\mathrm{MR}} \mathrm{Fp}^{\mathrm{S}} \mathrm{Pe}^{\mathrm{RS}} \mathrm{Fd}^{\mathrm{S}}$ & $\mathrm{H} 277 \mathrm{Y}$ & N.F. & N.F. \\
\hline 4-B48 & Unknown & & $>100$ & 0.01 & 4.69 & 1.84 & $\mathrm{Bo}^{\mathrm{HR}} \mathrm{Fp}^{\mathrm{S}} \mathrm{Pe}^{\mathrm{RS}} \mathrm{Fd}^{\mathrm{RS}}$ & $\mathrm{H} 277 \mathrm{Y}$ & N.F. & N.F. \\
\hline 4-B51 & Unknown & & $>100$ & 0.06 & 1.62 & 0.02 & $\mathrm{Bo}^{\mathrm{HR}} \mathrm{Fp}^{\mathrm{S}} \mathrm{Pe}^{\mathrm{RS}} \mathrm{Fd}^{\mathrm{S}}$ & $\mathrm{H} 277 \mathrm{Y}$ & N.F. & N.F. \\
\hline $4-B 52$ & Unknown & & $>100$ & 0.39 & 4.13 & 4.98 & $\mathrm{Bo}^{\mathrm{HR}} \mathrm{Fp}^{\mathrm{S}} \mathrm{Pe}^{\mathrm{RS}} \mathrm{Fd}^{\mathrm{RS}}$ & $\mathrm{H} 277 \mathrm{Y}$ & N.F. & N.F. \\
\hline 4-B53 & Unknown & & $>100$ & 0.01 & 58.26 & 0.38 & $\mathrm{Bo}^{\mathrm{HR}} \mathrm{Fp}^{\mathrm{S}} \mathrm{Pe}^{\mathrm{MR}} \mathrm{Fd}^{\mathrm{S}}$ & $\mathrm{H} 277 \mathrm{Y}$ & N.F. & N.F. \\
\hline 4-A25 & Unknown & & $>100$ & 0.73 & $>100$ & 91.07 & $\mathrm{Bo}^{\mathrm{HR}} \mathrm{Fp}^{\mathrm{S}} \mathrm{Pe}^{\mathrm{HR}} \mathrm{Fd}^{\mathrm{MR}}$ & N.F. & H134R & N.F. \\
\hline $4-\mathrm{A} 26$ & Unknown & & $>100$ & 0.18 & $>100$ & 3.71 & $\mathrm{Bo}^{\mathrm{HR}} \mathrm{Fp}^{\mathrm{S}} \mathrm{Pe}^{\mathrm{HR}} \mathrm{Fd}^{\mathrm{RS}}$ & N.F. & H134R & N.F. \\
\hline 4-A27 & Unknown & & $>100$ & 0.04 & 31.34 & $>100$ & $\mathrm{Bo}^{\mathrm{HR}} \mathrm{Fp}^{\mathrm{S}} \mathrm{Pe}^{\mathrm{MR}} \mathrm{Fd}^{\mathrm{HR}}$ & N.F. & H134R & N.F. \\
\hline $4-\mathrm{A} 28$ & Unknown & & $>100$ & 0.08 & 42.32 & 1.25 & $\mathrm{Bo}^{\mathrm{HR}} \mathrm{Fp}^{\mathrm{S}} \mathrm{Pe}^{\mathrm{MR}} \mathrm{Fd}^{\mathrm{RS}}$ & $\mathrm{H} 277 \mathrm{Y}$ & H134R & N.F. \\
\hline 4-A33 & Unknown & & $>100$ & 0.15 & N.D. & $>100$ & $\mathrm{Bo}^{\mathrm{HR}} \mathrm{Fp}^{\mathrm{S}} \mathrm{Pe}^{\mathrm{N} \cdot \mathrm{D}} \cdot \mathrm{Fd}^{\mathrm{HR}}$ & $\mathrm{H} 277 \mathrm{Y}$ & H134R & N.F. \\
\hline
\end{tabular}


boscalid and fluxapyroxad $(P=0.0005, r=0.34)$, penthiopyrad and fluxapyroxad $(P=0.0026, r=0.29)$, penthiopyrad and fluopy$\operatorname{ram}(P=0.0031, r=0.29)$, and fluxapyroxad and fluopyram $(P=$ $0.0078, r=0.26)$. There was no correlation between sensitivities to boscalid and fluopyram $(P>0.1, r=0.10)$.

Molecular diagnosis of boscalid-resistant phenotypes. In total, 69,1 , and 1 A. alternata isolates with high, moderate, and reduced sensitivity to boscalid, respectively, along with 11 boscalidsensitive isolates were further investigated for the presence of known boscalid-resistance-associated mutations in A. alternata $S D H B(A a S D H B), S D H C(A a S D H C)$, and SDH D (AaSDHD) genes using previously developed PCR assays. Of the 11 boscalidsensitive isolates, 9, as expected, did not carry any known mutations in $A a S D H B, A a S D H C$, or $A a S D H D$ genes and were designated as genotype 1 . Noticeably, three isolates highly resistant to boscalid (4-A43, 4-A38, and 4-A84) and the isolate with reduced sensitivity to boscalid (4-B31) were also designated as genotype 1 because they did not carry any known mutations in $A a S D H B$, $A a S D H C$, or $A a S D H D$ genes. Based on the presence of specific mutations in $A a S D H B, A a S D H C$, and $A a S D H D$ genes, the remaining 66 isolates highly resistant to boscalid and the isolate (4-B54) with moderate resistance to boscalid were separated in five different genotypes. In all, 47 of the 66 isolates highly resistant to boscalid and the isolate with moderate resistance to boscalid (4-B54) possessed the mutations leading to H277Y $(n=48)$ amino acid substitution in AaSDHB (SDHB-H277Y mutants) and were designated as genotype 2 . Two isolates highly resistant to boscalid (4B12 and 4-B19) had the H277R amino acid substitution in AaSDHB (SDHB-H277R mutants) and were designated as genotype 3. Fourteen isolates highly resistant to boscalid had a mutation leading to the H134R substitution in AaSDHC (SDHC-H134R mutants) and were designated as genotype 4 . Noticeably, two boscalid-sensitive isolates (4-B37 and 4-A79) also possessed the H134R substitution in AaSDHC and were also designated as genotype 4. Two isolates (4-A28 and 4-A33) carried both the SDHBH277Y and SDHC-H134R amino acid alterations and were designated as genotype 5. Finally, one isolate highly resistant to boscalid (4-A78) possessed a mutation leading to the H133R substitution in AaSDHD (SDHD-H133R mutant) and was designated as genotype 6. All the boscalid phenotypes and associated $S D H$ mutations are presented in Table 2.

Impacts of $S D H$ mutations on the fungicidal activities of fluopyram, penthiopyrad, and fluxapyroxad and their cross resistance patterns with boscalid. To assess the impact of $S D H$ mutations on the fungicidal activities of the new SDHIs fluopyram, penthiopyrad, and fluxapyroxad and their cross resistance with boscalid, the 82 boscalid-resistant and -sensitive isolates in the respective genotype ( 1 to 5), as designated above, were also tested for their sensitivities against the new SDHI fungicides fluopyram, penthiopyrad, and fluxapyroxad.
All the isolates in the genotype 1 group of isolates, including the nine boscalid-sensitive isolates $\left(2<R_{\mathrm{f}}<60\right)$ and the three $(4-\mathrm{A} 43$, 4-A38, and 4-A84) and one (4-B31) mutants with high resistance $\left(R_{\mathrm{f}}>10,000\right)$ and reduced sensitivity $\left(R_{\mathrm{f}}=401\right)$ to boscalid, respectively, were found to be sensitive to fluopyram. The $R_{\mathrm{f}}$ values were 10 to 660 (Table 3). Of the nine boscalid-sensitive isolates in genotype 1 , eight were also sensitive to penthiopyrad $\left(1.5<R_{\mathrm{f}}<\right.$ 12.5). Two isolates highly resistant to boscalid (4-A38 and 4-A84; $\left.R_{\mathrm{f}}>10,000\right)$ were also highly resistant to penthiopyrad $\left(R_{\mathrm{f}}>\right.$ $5,000)$, whereas isolate $4-\mathrm{A} 43$ remained sensitive to this fungicide $\left(R_{\mathrm{f}}=6.5\right.$; Table 3$)$. Isolates $4-\mathrm{A} 43$ and $4-\mathrm{A} 84$ and seven boscalidsensitive isolates were sensitive to fluxapyroxad $\left(1<R_{\mathrm{f}}<130\right)$, whereas two isolates (4-A17 and 4-A90) were highly sensitive to this fungicide $\left(R_{\mathrm{f}}=1\right.$; Table 3$)$.

The 48 boscalid-resistant isolates $\left(R_{\mathrm{f}}=6,151\right.$ and $\left.>10,000\right)$ in the designated genotype 2 (SDHB-H277Y mutants) showed different levels of sensitivity to fluopyram. The $R_{\mathrm{f}}$ values were 1 to 480 (Table 3). These 48 boscalid-resistant isolates in genotype 2 displayed different levels of sensitivity and cross resistance with penthiopyrad, with $R_{\mathrm{f}}$ values of 2 to 5,000. For instance, the boscalid-resistant mutants 4-A60, 4-A72, 4-A44, 4-B43, 4-A51, and 4-A61 had high levels of resistance to boscalid $\left(R_{\mathrm{f}}>10,000\right)$ but their $R_{\mathrm{f}}$ value against penthiopyrad was $9,29,88,656,2,294$, and $>5,000$, respectively. Similarly, the 48 boscalid-resistant isolates in genotype 2 displayed different sensitivity and cross resistance patterns to fluxapyroxad. The $R_{\mathrm{f}}$ values were 20 to $>100,000$ (Table 3 ). For instance, the boscalid-resistant mutants 4-A16, 4-A51, 4A39, 4-A36, 4-B43, and 4-A72 had high levels of resistance to boscalid $\left(R_{\mathrm{f}}>10,000\right)$ but their $R_{\mathrm{f}}$ value against fluxapyroxad was $20,90,890,3,260,22,060$, and $>100,000$, respectively.

Concerning the two SDHB-H277R mutants (4-B12 and 4-B19) in genotype 3 , which were mutants highly resistant to boscalid $\left(R_{\mathrm{f}}\right.$ $>10,000)$, both showed sensitivity to fluopyram $\left(R_{\mathrm{f}}=100\right.$ and 120 , respectively), penthiopyrad $\left(R_{\mathrm{f}}=44\right.$ for 4-B12), and fluxapyroxad $\left(R_{\mathrm{f}}=20\right.$ and 60 , respectively) (Table 3 ).

The 14 isolates highly resistant to boscalid, designated as SDHC-H134R mutants (genotype 4), displayed different sensitivity and levels of cross resistance to fluopyram. The $R_{\mathrm{f}}$ values were 20 to 22,900 (Table 3). For instance, the boscalid-resistant mutants 4A49, 4-A56, 4-B18, 4-A81, and 4-B20 had high level of resistance to boscalid $\left(R_{\mathrm{f}}>10,000\right)$ but their $R_{\mathrm{f}}$ value against fluopyram was $20,290,1,520,2,240$, and 22,900, respectively. Similarly, these type-4-genotype mutants showed different patterns of sensitivity and cross resistance to penthiopyrad and fluxapyroxad. The $R_{\mathrm{f}}$ values were 112 to 5,000 , and 1 to $>100,000$ for penthiopyrad and fluxapyroxad, respectively (Table 3). The two SDHC-H134R mutants (4-B37 and 4-A79), which were sensitive to boscalid $\left(R_{\mathrm{f}}=9\right.$ and 80, respectively), were also sensitive to fluopyram $\left(R_{\mathrm{f}}=30\right.$ and 50 , respectively), penthiopyrad $\left(R_{\mathrm{f}}=11\right.$ and 21.5 , respectively), and fluxapyroxad $\left(R_{\mathrm{f}}=10\right.$ and 160 , respectively) (Table 3$)$.

Table 3. Impacts of known amino acid alterations in succinate dehydrogenase (SDH) subunits on the levels of cross sensitivity or resistance between succinate dehydrogenase inhibitor (SDHI) fungicides in Alternaria alternata isolates collected in 2010 from several commercial pistachio orchards with a history of boscalid usage

\begin{tabular}{|c|c|c|c|c|c|c|}
\hline \multirow[b]{2}{*}{$N^{\mathbf{b}}$} & \multicolumn{2}{|c|}{ Genotype } & \multicolumn{4}{|c|}{$R_{\mathrm{f}}$ range $^{\mathrm{a}}$} \\
\hline & Category & Alterationc $^{c}$ & Boscalid & Fluopyram & Penthiopyrad & Fluxapyroxad \\
\hline 9 & 1 & None & $2-60$ & $50-570$ & $1.5-12.5$ & $1-130$ \\
\hline 3 & $\ldots$ & $\ldots$ & $>10,000$ & $10-470$ & $6.5-5,000$ & $10-40$ \\
\hline 1 & $\ldots$ & $\ldots$ & 401 & 660 & 50.5 & 1,400 \\
\hline 48 & 2 & SDHB-H277Y & $6,151 \rightarrow>10,000$ & $1-480$ & $2-5,000$ & $20->100,000$ \\
\hline 2 & 3 & SDHB-H277R & $>10,000$ & $100-120$ & 44 & $20-60$ \\
\hline 2 & 4 & SDHC-H134R & $9-80$ & $30-50$ & $11-21.5$ & $10-160$ \\
\hline 14 & $\ldots$ & SDHC-H134R & $>10,000$ & $20-22,900$ & $112-5,000$ & $1->100,000$ \\
\hline 2 & 5 & SDHB-H277Y & $\ldots$ & $\ldots$ & $\ldots$ & $\ldots$ \\
\hline & $\ldots$ & SDHC-H134R & $>10,000$ & $80-150$ & 2,116 & $1,250->100,000$ \\
\hline 1 & 6 & SDHD-H133R & $>10,000$ & 2,930 & 245 & $>100,000$ \\
\hline
\end{tabular}

${ }^{\text {a }}$ Resistance factor $\left(R_{\mathrm{f}}\right)=50 \%$ effective concentration $\left(\mathrm{EC}_{50}\right)$ value of a particular isolate for a specific fungicide divided by the lowest EC $\mathrm{E}_{50}$ value.

b Number of isolates.

c Amino acid alteration in SDH subunits. 
The two mutants (4-A28 and 4-A33), which carried both the SDHB-H277Y and SDHC-H134R alterations (genotype 5), were sensitive to fluopyram $\left(R_{\mathrm{f}}=80\right.$ and 150 , respectively) but showed cross resistance to penthiopyrad $\left(R_{\mathrm{f}}=2,116\right.$ for 4-A28) and fluxapyroxad $\left(R_{\mathrm{f}}=1,250\right.$ for 4-A28 and $>100,000$ for 4-A33).

Finally, the SDHD-H133R mutant (4-A78), which was highly resistant to boscalid $\left(R_{\mathrm{f}}>10,000\right)$ and designated as genotype 6 , showed cross resistance to fluopyram $\left(R_{\mathrm{f}}=2,930\right)$ and fluxapyroxad boscalid $\left(R_{\mathrm{f}}>100,000\right)$ but a lower $R_{\mathrm{f}}$ of 245 was obtained for penthiopyrad (Table 3 ).

Frequencies of SDHIs resistance in pistachio orchards. Boscalid resistance was detected in all the pistachio orchards. Overall, the frequency of phenotypes highly resistant to boscalid was predominant in the A. alternata population. The two highest frequencies for boscalid resistance were observed in pistachio orchards located in Madera (84.4\%) and at an unknown location $(88.9 \%)$ (Table 4). Different phenotypes were observed for penthiopyrad and fluxapyroxad and at different proportions. By comparison, the frequencies of isolates highly resistant to penthiopyrad and fluxapyroxad were lower, 18.7 and $12.5 \%$, respectively, in Madera, whereas they were 16.7 and $11.1 \%$ for penthiopyrad and fluxapyroxad, respectively, at the unknown location (Table 4). There were no isolates highly resistant to fluopyram (Table 4). The SDHB mutations were the most prevalent in the A. alternata boscalidresistant populations at the various locations (Table 4).

\section{Discussion}

The performance of boscalid-containing product for controlling ALB of pistachio has been seriously reduced due to the development of A. alternata populations resistant to boscalid, concomitantly with the widespread occurrence of Alternaria strobilurinresistant isolates across California pistachio orchards $(1-4,14)$. Recently, new SDHI fungicides such as fluopyram, penthiopyrad, and fluxapyroxad have been introduced into the marketplace. For new chemical fungicides, establishment of baseline sensitivity of plant pathogens against which future changes can be measured is essential for estimating the risk of resistance development against particular chemical groups of fungicides (21). In this study, we first established the baseline sensitivity profiles to penthiopyrad, fluopyram, and fluxapyroxad of A. alternata isolates originating from commercial pistachio orchards that had never been exposed to boscalid or any of the new SDHIs and compared them with boscalid sensitivity data obtained for the same isolates. Correlation analyses indicated that there were significant positive baseline sensitivity relationships between boscalid and fluxapyroxad sensitivities and fluxapyroxad and penthiopyrad sensitivities, whereas no other relationships were significant. Differential sensitivity and positive or lack of correlation among baseline population of fungal pathogens to several SDHI fungicides have been observed in other studies $(9,10,26,27)$. Further comparison of the sensitivity values obtained for sensitive isolates from both baseline- and boscalid- exposed populations showed that boscalid had significantly lower activity against $A$. alternata than the new benzamide and pyrazole carboxamides.

Another important objective of this study was to assess the potential impact of boscalid resistance and associated mutations on the performance of the new SDHIs and to get more insight in their cross resistance patterns with boscalid. Results of sensitivity tests for several field isolates originating from commercial orchards that have been exposed to only boscalid showed that phenotypes highly resistant to boscalid were prevalent in the sampled A. alternata population. Molecular investigation of the occurrence of known $S D H$-mutations in the boscalid-resistant phenotypes showed the occurrence of different genotypes based upon the presence of mutations in their $S D H$ target genes. A. alternata boscalid-resistant mutants mainly harbored amino acid substitutions in subunits AaSDHB (H277Y and H277R) (genotype 2 and genotype 3) (5) and AaSDHC (H134R) (genotype 4) (6), with H277Y being predominant, as previously reported $(3,5,6)$. Only one isolate had the H133R change in AaSDHD (genotype 6) (6). Two mutants were found to carry both the SDHB-H277Y and SDHC-H134R alterations and they were designated as genotype 5. The sensitivities to fluopyram, penthiopyrad, and fluxapyroxad of the described genotypes were then assessed and, depending upon the fungicide and the attributed genotype, different sensitivities and levels of crossresistance between boscalid and the new SDHIs were observed.

The A. alternata boscalid-resistant isolates displaying the SDHB-H277Y alteration (genotype 2) showed low, moderate, or high levels of resistance to penthiopyrad. Similarly, some boscalidresistant mutants with SDHB-H277Y (genotype 2) showed low, moderate, or high levels of resistance to fluxapyroxad. The obtained results demonstrated that the SDHB-H277Y alteration conferred a positive cross-resistance pattern between boscalid and the SDHIs penthiopyrad and fluxapyroxad, although lower $R_{\mathrm{f}} \mathrm{s}$ for penthiopyrad and fluxapyroxad were observed for some mutants. These results are in agreement with several previous studies that revealed differential levels of cross resistance within and between structurally different SDHI fungicides in diverse uncharacterized or SDHB-H277Y mutants of several other plant-pathogenic fungi $(3,7,12,13,28,29)$. Interestingly, the results also showed that some SDHB-H277Y boscalid-resistant mutants (genotype 2) were sensitive to penthiopyrad. Similarly, some SDHB-H277Y boscalidresistant mutants were sensitive to fluxapyroxad. These results are in agreement with those from a recent study showing that $A$. alternata isolates from pistachio with SDHB-H277Y alteration were resistant to boscalid but few mutants with this genotype were still sensitive to boscalid (23). The absence of cross-resistance between boscalid and penthiopyrad among uncharacterized isolates that have developed resistance to boscalid or SDHB-H277Y mutants have been reported in $A$. solani $(10,17)$. The lack of crossresistance among some A. alternata SDHB-H277Y mutants when comparing their sensitivity of boscalid-resistant isolates to

Table 4. Frequencies of succinate dehydrogenase (SDH) inhibitor resistance and SDH mutations in Alternaria alternata SDH mutants detected in California pistachio orchards

\begin{tabular}{|c|c|c|c|c|c|c|c|c|c|c|c|c|c|c|c|c|c|c|c|c|c|}
\hline \multirow[b]{3}{*}{ City } & \multirow[b]{3}{*}{$N^{\mathrm{d}}$} & \multicolumn{16}{|c|}{ Frequency $(\%)$ of fungicide resistance ${ }^{a}$} & \multirow[b]{3}{*}{$S D H(N)^{\mathrm{e}}$} & \multirow{2}{*}{\multicolumn{3}{|c|}{ Frequency of $S D H(\%)^{b}$}} \\
\hline & & \multicolumn{4}{|c|}{ Boscalid } & \multicolumn{4}{|c|}{ Fluopyram } & \multicolumn{4}{|c|}{ Penthiopyrad } & \multicolumn{4}{|c|}{ Fluxapyroxad } & & & & \\
\hline & & HR & MR & $\mathbf{L R}$ & $\mathbf{R S}$ & HR & MR & LR & $\mathbf{R S}$ & HR & MR & LR & $\mathbf{R S}$ & HR & MR & LR & RS & & $S D H B$ & $S D H C$ & $S D H D$ \\
\hline Parlie & 8 & 12.5 & 0 & 0 & 12.5 & 0 & 0 & 0 & 0 & 0 & 0 & 0 & 0 & 12.5 & 0 & 0 & 0 & 1 & 100 & 0 & 0 \\
\hline Terrabella & 12 & 16.7 & 0 & 0 & 8.3 & 0 & 0 & 0 & 0 & 0 & 16.7 & 0 & 0 & 0 & 16.7 & 0 & 0 & 2 & 50 & 50 & 0 \\
\hline Corcoran & 7 & 14.3 & 0 & 0 & 14.3 & 0 & 0 & 0 & 0 & 14.3 & 0 & 0 & 14.3 & 14.3 & 14.3 & 0 & 14.3 & 2 & 50 & 50 & 0 \\
\hline Madera & 32 & 84.4 & 0 & 0 & 0 & 0 & 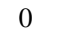 & 0 & 6.2 & 18.7 & 21.9 & 12.5 & 9.4 & 12.5 & 7.7 & 3.1 & 18.7 & 25 & 76 & 24 & 0 \\
\hline Hanford & 14 & 42.9 & 0 & 0 & 0 & 0 & 0 & 0 & 7.1 & 14.3 & 14.3 & 7.1 & 0 & 0 & 14.3 & 7.1 & 7.1 & 6 & 83.3 & 16.7 & 0 \\
\hline Shafter & 26 & 61.5 & 0 & 0 & 0 & 0 & 3.8 & 0 & 15.4 & 23.0 & 19.2 & 0 & 7.7 & 15.4 & 7.7 & 0 & 7.7 & 15 & 73.33 & 20.0 & 6.67 \\
\hline Unknown & 18 & 88.9 & 5.5 & 0 & 0 & 0 & 0 & 0 & 0 & 16.7 & 22.2 & 5.5 & 33.3 & 11.1 & 16.7 & 0 & 27.8 & 17 & 70.6 & 29.4 & 0 \\
\hline
\end{tabular}

${ }^{a} \mathrm{RS}=$ reduced sensitivity, $\mathrm{LR}=$ low resistance, $\mathrm{MR}=$ moderately resistant, and $\mathrm{HR}=$ highly resistant.

b Frequency of $S D H$ mutations.

c Orchard locations.

d Number of isolates.

e Number of $S D H$ mutations. 
penthiopyrad and fluxapyroxad indicates that described H277Y mutations in the SDHB subunit that confer resistance to boscalid (5) do not always confer resistance to penthiopyrad and fluxapyroxad and that their binding site on complex II may slightly differ from that of boscalid (22). This also means that additional amino acid residues at different codons in SDH subunits are targeted by the latter two fungicides and, thus, additional mechanisms of resistance are likely involved. Two A. alternata boscalid-resistant isolates that displayed the SDHB-H277R mutations (genotype 3) were sensitive to penthiopyrad and fluxapyroxad. The absence or lack of cross-resistance between the SDHI fungicides boscalid and penthiopyrad among some SDHBH277R mutants that have developed resistance to boscalid have been reported in other fungi $(7,10)$. Lack of cross resistance between boscalid and fluxapyroxad among some SDHB-H277R mutants that have developed resistance to boscalid have been reported in Botrytis cinerea (28). The obtained results indicate that the described H277R amino acid substitution in the SDHB subunit that confer resistance to boscalid do not always affect the binding and, thus, the fungicidal activities of penthiopyrad and fluxapyroxad (7). Regarding the fungicidal activity of fluopyram on the boscalid-resistant isolates possessing the SDHB-H277Y (genotype 2) and SDHB-H277R (genotype 3) alterations, our results showed that all these $S D H B$ genotypes remained sensitive to fluopyram. Lack of cross resistance between boscalid and fluopyram in boscalid-resistant mutants carrying $S D H B$ mutations has been reported in several other fungal species $(7,10,12$, 22,28). This indicates that the described H277Y and H277R amino acid substitutions in the SDHB subunit that confer resistance to boscalid do not affect but rather increase the binding of fluopyram and, thus, its fungicidal activity, and this may be linked to the molecular structure of this benzamide derivative and its interaction with $\operatorname{SDHB}(5,12,13,28)$. Amino acid alterations at similar or different codons in the SDHB subunit have been shown to confer low to moderate levels of resistance to fluopyram in isolates of $B$. cinerea or Mycosphaerella graminicola moderately or highly resistant to boscalid $(22,28)$, and the existence of such SDHB alterations in yet-to-be-discovered fluopyram-resistant phenotypes in A. alternata from pistachio orchards cannot be excluded.

Some A. alternata boscalid-resistant SDHC-H134R mutants (genotype 4) showed low, moderate, and high levels of resistance to penthiopyrad. Similarly, some boscalid-resistant SDHC-H134R mutants (genotype 4) showed low, moderate, or high levels of resistance to fluxapyroxad. The obtained results demonstrated that the SDHC-H134R alteration can confer a positive cross-resistance pattern between boscalid and the SDHIs penthiopyrad and fluxapyroxad, although lower $R_{\mathrm{f}} \mathrm{s}$ were observed for penthiopyrad and fluxapyroxad in some mutants. These results are in agreement with other previous studies that revealed differential levels of cross resistance between structurally different SDHI fungicides in SDHC mutants of some plant-pathogenic fungi $(3,22)$. Analysis of the effect of fluopyram on the 14 A. alternata SDHC-H134R mutants that were resistant to boscalid showed that three and one SDHCH134R mutants had reduced sensitivity and moderately resistant phenotypes, respectively, to fluopyram. In contrast to a previous study, where we reported that boscalid-resistant isolates with the H134R-SDHC alteration expressed low levels of resistance to fluopyram (3), it is clear here that this alteration also confers moderate levels of resistance to fluopyram. Positive cross resistance between boscalid and fluopyram in SDHC mutants of fungal species has been previously reported $(3,22)$. Curiously, among the $14 \mathrm{~A}$. alternata SDHC mutants that were resistant to boscalid, several remained sensitive to fluopyram. These results are in agreement with those from a recent study showing that $A$. alternata isolates from pistachio with the SDHB-H134R alteration were resistant to boscalid but few mutants with this genotype were still sensitive to boscalid (23). Some A. solani boscalid-resistant isolates with the SDHC-H134R substitution were found to be sensitive to penthiopyrad (17). Our results further showed that two $A$. alternata isolates that had the H134R alteration in the SDHC subunit remained sensitive not only to fluopyram but also to the three other SDHI fungicides. The absence and discrepancies of cross-resistance patterns between boscalid and fluopyram observed in the SDHC mutants suggest that the SDHC-H134R amino acid substitutions that confer resistance to boscalid (6) do not always result in a loss of sensitivity to fluopyram. This further implies that the fluopyram binding site in complex II may slightly differ from that of boscalid and that there might be additional amino acid residues in A. alternata SDH subunits in these mutants that are potentially or specifically targeted by fluopyram, thus resulting in a fluopyram-sensitive phenotype. This also supports the existence of additional mechanisms of resistance (22).

In this study, only one A. alternata isolate carried the SDHDH133R alteration (genotype 6). The low frequency of $A$. alternata isolates carrying this type of mutation could be explained by the fitness penalty associated with SDHD mutants (6). This SDHD mutant showed cross resistance between boscalid and the new SDHIs but lower $R_{\mathrm{f}} \mathrm{S}$ were observed for fluopyram and penthiopy$\mathrm{rad}$. These results are in agreement with several previous studies that revealed differential levels of cross resistance between structurally different SDHI fungicides in diverse SDHD mutants of some plant-pathogenic fungi $(3,17,22)$.

In summary, the research presented here provided some critical information about the frequencies of boscalid-resistant isolates in A. alternata populations from pistachio orchards at several locations in California and allowed us to establish the baseline sensitivity data to new SDHIs, study their cross-resistance relationships with boscalid, and assess the impact of SDH mutations on their performance. Overall, the baseline sensitivity data and the lack and the different levels of cross resistance between SDHIs presented here will have important implications for the rational and sustained deployment of the new SDHI fungicides into ALB fungicide spray programs. Even though the boscalid-resistant mutants were sensitive or displayed reduced sensitivity or low, moderate, or high resistance to penthiopyrad and fluxapyroxad, it is expected that the performance of these two fungicides will eventually be affected because mutants with full cross resistance to these fungicides will be selected and will eventually prevail in situations of prolonged SDHI exposure, while the sensitive ones will be eliminated $(21,27)$. In light of these findings, when penthiopyrad and fluxapyroxad fungicides become registered in pistachio, they should not be used in the same spray program or in pistachio orchards where resistance to boscalid is established. Regarding fluopyram, the lack of cross resistance observed between boscalid and fluopyram, particularly in the case of the SDHB mutants, seems consistent with the excellent efficacy provided thus far by fluopyram against ALB as observed in experimental orchards where Luna products have been used or in commercial orchards where high levels of boscalid resistance in A. alternata have been detected ( H. F. Avenot and T. J. Michailides, unpublished data). However, because of the detection in this study of A. alternata SDHC and SDHD mutants showing cross resistance between boscalid and fluopyram, the use of these two SDHI fungicides in the same spray program or in pistachio orchards where resistance to boscalid is established should also be avoided. Continuous monitoring of variation in fluopyram sensitivity in A. alternata populations from pistachio orchards where growers have started to apply Luna products will be required and this might lead to the detection of additional $A$. alternata fluopyram-resistant phenotypes. Subsequent sequencing of $S D H$ genes in such phenotypes could lead to the characterization of unknown mutations potentially associated with resistance. Information that will be generated from such monitoring studies will also help determine which commercial pistachio orchards will be at risk for resistance development and will subsequently help to cope with eventual fluopyram resistance problems by developing applicable antiresistance management strategies. Future studies involving inoculation of isolates with different phenotypes or genotypes onto detached pistachio leaves will also assess their fitness and impact on disease control. 


\section{Acknowledgments}

We thank the California Pistachio Research Board for their financial support and C. Solorio for technical assistance.

\section{Literature Cited}

1. Avenot, H. F., and Michailides, T. J. 2007. Resistance to boscalid fungicide in Alternaria alternata isolates from pistachio in California. Plant Dis. 91:1345-1350.

2. Avenot, H. F., and Michailides, T. J. 2009. Monitoring the sensitivity to boscalid in Alternaria alternata populations from California pistachio orchards. (Abstr.) Phytopathology 99:S6.

3. Avenot, H. F., and Michailides, T. J. 2010. Progress in understanding molecular mechanisms and evolution of resistance to succinate dehydrogenase inhibiting (SDHI) fungicides in phytopathogenic fungi. Crop Prot. 29:643-651.

4. Avenot, H., Morgan, D., and Michailides, T. J. 2008. Resistance to pyraclostrobin, boscalid and multiple resistance to Pristine (pyraclostrobin + boscalid) fungicide in Alternaria alternata causing alternaria late blight of pistachios in California. Plant Pathol. 57:135-140.

5. Avenot, H. F., Sellam, A., Karaoglanidis, G., and Michailides, T. J. 2008. Characterization of mutations in the iron sulfur subunit of succinate dehydrogenase correlating with boscalid resistance in Alternaria alternata from California pistachio. Phytopathology 98:736-742.

6. Avenot, H., Sellam, A., and Michailides, T. J. 2009. Characterization of mutations in the membrane-anchored subunits AaSDHC and AaSDHD of succinate dehydrogenase from Alternaria alternata isolates conferring field resistance to the fungicide boscalid. Plant Pathol. 58:1134-1143.

7. Avenot, H. F., Thomas, A., Gitaitis, R. D., Langston Jr., D. B., and Stevenson, K. 2012. Molecular characterization of boscalid- and penthiopyrad-resistant isolates of Didymella bryoniae and assessment of their sensitivity to fluopyram. Pest Manage. Sci. 68:645-651.

8. Brent, K. J. 1995. Fungicide Resistance in Crop Pathogens: How Can it be Managed? GIFAP, Brussels.

9. Fraaije, B. A., Bayon, C., Atkins, S., Cools, H. J., Lucas, J. A., and Fraaije, M. W. 2012. Risk assessment studies on succinate dehydrogenase inhibitors, the new weapons in the battle to control Septoria leaf blotch in wheat. Mol. Plant Pathol. 13:263-275.

10. Gudmestad, N., Arabiat, S., Miller, J., and Pasche, J. 2013. Prevalence and impact of SDHI fungicide resistance in Alternaria solani. Plant Dis. 97:952-960.

11. Horsefield, R., Yankovskaya, V., Sexton, G., Whittingham, W., Shiomi, K., Omura. S., Byrne, B., Cecchini, G., and Iwata, S. 2006. Structural and computational analysis of the quinone-binding site of complex II (succinateubiquinone oxidoreductase): A mechanism of electron transfer and proton conduction during ubiquinone reduction. J. Biol. Chem. 281:7309-7316.

12. Ishii, H., Miyamoto, T., Ushio, S., and Kakishima, M. 2011. Lack of crossresistance to a novel succinate dehydrogenase inhibitor, fluopyram, in highly boscalid-resistant isolates of Corynespora cassiicola and Podosphaera xanthii. Pest Manage. Sci. 67:474-482.

13. Leroux, P., Gredt, M., Leroch, M., and Walker, A. S. 2010. Exploring mechanisms of resistance to respiratory inhibitors in field strains of Botrytis cinerea, the causal agent of grey mold. Appl. Environ. Microbiol. 76:6615-6630.

14. Luo, Y., Ma, Z., Reyes, H. C., Morgan, D. P., and Michailides, T. J. 2007. Using real-time PCR to survey frequency of azoxystrobin-resistant allele
G143A in Alternaria populations from almond and pistachio orchards in California. Pestic. Biochem. Physiol. 88:328-336.

15. Ma, Z., Felts, D., and Michailides, T. J. 2003. Resistance to azoxystrobin in Alternaria isolates from pistachio in California. Pestic. Biochem. Physiol. 77:66-74.

16. Ma, Z., and Michailides, T. J. 2004. A real-time PCR assay for detection of azoxystrobin-resistant Alternaria populations from pistachio orchards in California. Crop Prot. 223:1259-1263.

17. Mallik, I., Arabiat, S., Pasche, J., Bolton, M. D., Patel, J. S., and Gudmestad, N. C. 2014. Molecular characterization and detection of mutations associated with resistance to succinate dehydrogenase-inhibiting fungicides in Alternaria solani. Phytopathology 104:40-49.

18. Michailides, T. J., Morgan, D. P., Ma, Z., Luo, Y., Felts, D., Doster, M. A., and Reyes, H. 2005. Conventional and molecular assays aid diagnosis of crop diseases and fungicide resistance. Calif. Agric. 59:115-123.

19. Miyamoto, T., Ishii, H., Stammler, G., Koch, A., Ogawara, T., Tomita, Y., Fountaine, J. M., Ushio, S., Seko, T., and Kobori, S. 2010. Distribution and molecular characterization of Corynespora cassiicola isolates resistant to boscalid. Plant Pathol. 59:873-881.

20. Pryor, B., and Michailides, T. J. 2002. Morphological, pathogenic, and molecular characterization of Alternaria isolates associated with Alternaria late blight of pistachio. Phytopathology 92:406-416.

21. Russel, P. E. 2004. Sensitivity baselines in fungicide resistance research and management. FRAC, Monogr. No. 3, Brussels.

22. Scalliet, G., Bowler, J., Luksch, T., Kirchhofer-Allan, L., Steinhauer, D., Ward, K., Niklaus, M., Verras, A., Csukai, M., Antoine Daina, A., and Fonne-Pfister, R. 2012. Mutagenesis and functional studies with succinate dehydrogenase inhibitors in the wheat pathogen Mycosphaerella graminicola. PLoS One 7(4):e35429. Online publication. doi:10.1371/journal. pone.0035429

23. Sierotzki, H., Frey, R., Morchoisne, M., Olaya, G., Mösch, M., and Scalliet, G. 2011. Sensitivity of fungal pathogens to SDHI fungicides. Pages 179186 in: Modern Fungicides and Antifungal Compounds VI. H. W. Dehne, H. B. Deising, U. Gisi, K. H. Kuck, P. E. Russell, et al. eds. DPG-Verlag, Braunschweig, Germany.

24. Stammler, G., Brix, H. D., Nave, B., Gold, R., and Schoefl, U. 2008. Studies on the biological performance of boscalid and its mode of action. Pages 45-51 in: Modern Fungicides and Antifungal Compounds V. H. W. Dehne, H. B. Deising, U. Gisi, K. H. Kuck, P. Russell, and H. Lyr, eds. DPG, Braunschweig, Germany.

25. Stamm.er, G., and Speakman, J. 2006. Microtiter method to test the sensitivity of Botrytis cinerea to boscalid. J. Phytopathol. 154:508-510.

26. Thomas, A., Langston, D. B., Jr., and Stevenson, K. L. 2012. Baseline sensitivity and cross-resistance to succinate-dehydrogenase-inhibiting and demethylation-inhibiting fungicides in Didymella bryoniae. Plant Dis. 96:979-984.

27. Veloukas, T., and Karaoglanidis, G. S. 2012. Biological activity of the succinate dehydrogenase inhibitor fluopyram against Botrytis cinerea and fungal baseline sensitivity. Pest Manage. Sci. 68:858-864.

28. Veloukas, T., Markoglou, and Karaoglanidis, G. S. 2013. Differential effect of $S d h \mathrm{~B}$ gene mutations on the sensitivity to SDHI fungicides in Botrytis cinerea. Plant Dis. 97:118-122.

29. Yin, Y. N., Kim, Y. K., and Xiao, C. L. 2011. Molecular characterization of boscalid resistance in field isolates of Botrytis cinerea from apple. Phytopathology 101:986-995. 